\title{
Isoparametric and Dupin Hypersurfaces ${ }^{\star}$
}

Thomas E. CECIL

Department of Mathematics and Computer Science, College of the Holy Cross, Worcester, MA 01610, USA

E-mail: cecil@mathcs.holycross.edu

URL: http://mathcs.holycross.edu/faculty/cecil.html

Received June 24, 2008, in final form August 28, 2008; Published online September 08, 2008

Original article is available at http://www.emis.de/journals/SIGMA/2008/062/

\begin{abstract}
A hypersurface $M^{n-1}$ in a real space-form $\mathbf{R}^{n}, S^{n}$ or $H^{n}$ is isoparametric if it has constant principal curvatures. For $\mathbf{R}^{n}$ and $H^{n}$, the classification of isoparametric hypersurfaces is complete and relatively simple, but as Élie Cartan showed in a series of four papers in 1938-1940, the subject is much deeper and more complex for hypersurfaces in the sphere $S^{n}$. A hypersurface $M^{n-1}$ in a real space-form is proper Dupin if the number $g$ of distinct principal curvatures is constant on $M^{n-1}$, and each principal curvature function is constant along each leaf of its corresponding principal foliation. This is an important generalization of the isoparametric property that has its roots in nineteenth century differential geometry and has been studied effectively in the context of Lie sphere geometry. This paper is a survey of the known results in these fields with emphasis on results that have been obtained in more recent years and discussion of important open problems in the field.
\end{abstract}

Key words: isoparametric hypersurface; Dupin hypersurface

2000 Mathematics Subject Classification: 53C40; 53C42; 53B25

\section{Introduction}

A hypersurface $M^{n-1}$ immersed in Euclidean space $\mathbf{R}^{n}$, the sphere $S^{n}$ or hyperbolic space $H^{n}$ is called isoparametric if it has constant principal curvatures. An isoparametric hypersurface in $\mathbf{R}^{n}$ can have at most two distinct principal curvatures, and it must be an open subset of a hyperplane, hypersphere or a spherical cylinder $S^{k} \times \mathbf{R}^{n-k-1}$. This was first proven for $n=3$ by Somigliana [134] in 1919 (see also B. Segre [128] and Levi-Civita [75]) and for arbitrary $n$ by B. Segre [129] in 1938. A similar result holds in $H^{n}$. However, as Élie Cartan $[8,9,10,11]$ showed in a series of four papers published in the period 1938-1940, the theory of isoparametric hypersurfaces in the sphere $S^{n}$ is much more beautiful and complicated.

Cartan produced examples of isoparametric hypersurfaces in spheres with $g=1,2,3$ or 4 distinct principal curvatures, and he classified those with $g \leq 3$. Approximately thirty years later, Münzner [93, 94] wrote two papers which greatly extended Cartan's work, proving that all isoparametric hypersurfaces are algebraic and that the number $g$ of distinct principal curvatures must be 1, 2, 3, 4 or 6 . Since Cartan had classified isoparametric hypersurfaces with $g \leq 3$, the classification of those with $g=4$ or 6 quickly became the goal of researchers in the field after Münzner's work. This classification problem has proven to be interesting and difficult, and it was listed as Problem 34 on Yau's [170] list of important open problems in geometry in 1990. The problem remains open in both cases $g=4$ and 6 at this time, although much progress has been made.

\footnotetext{
*This paper is a contribution to the Special Issue "Élie Cartan and Differential Geometry". The full collection is available at http://www.emis.de/journals/SIGMA/Cartan.html
} 
A hypersurface $M^{n-1}$ in one of the real space-forms $\mathbf{R}^{n}, S^{n}$ or $H^{n}$ is proper Dupin if the number $g$ of distinct principal curvatures is constant on $M^{n-1}$, and each principal curvature function is constant along each leaf of its corresponding principal foliation. This is clearly a generalization of the isoparametric condition, and it has its roots in nineteenth century differential geometry. Aside from the isoparametric surfaces in $\mathbf{R}^{3}$, the first examples of Dupin hypersurfaces are the cyclides of Dupin in $\mathbf{R}^{3}$. These are all surfaces that can be obtained from a torus of revolution, a circular cylinder or a circular cone by inversion in a 2 -sphere in $\mathbf{R}^{3}$. The cyclides have several other characterizations that will be discussed in this paper (see also [38, pp. 151-166], [26, pp. 148-159], [115]).

The proper Dupin property is preserved under Möbius (conformal) transformations, and an important class of compact proper Dupin hypersurfaces in $\mathbf{R}^{n}$ consists of those hypersurfaces that are obtained from isoparametric hypersurfaces in a sphere $S^{n}$ via stereographic projection from $S^{n}-\{P\}$ to $\mathbf{R}^{n}$, where $P$ is a point in $S^{n}$. Indeed several of the major classification results for compact proper Dupin hypersurfaces involve such hypersurfaces.

An important step in the theory of proper Dupin hypersurfaces was the work of Pinkall [112, 113, 114, 115] which situated the study of Dupin hypersurfaces in the setting of Lie sphere geometry. Among other things, Pinkall proved that the proper Dupin property is invariant under the group of Lie sphere transformations, which contains the group of Möbius transformations as a subgroup. The theory of proper Dupin hypersurfaces has both local and global aspects to it, and many natural problems remain open.

In this paper, we survey the known results for isoparametric and proper Dupin hypersurfaces with emphasis on results that have been obtained in more recent years, and discuss important open problems in the field. The reader is also referred to the excellent survey article by Thorbergsson [157] published in the year 2000.

\section{Isoparametric hypersurfaces}

Let $\tilde{M}^{n}(c)$ be a simply connected, complete Riemannian manifold of dimension $n$ with constant sectional curvature $c$, that is, a real space-form. For $c=0,1,-1$, respectively, $\tilde{M}^{n}(c)$ is Euclidean space $\mathbf{R}^{n}$, the unit sphere $S^{n} \subset \mathbf{R}^{n+1}$, or hyperbolic space $H^{n}$. According to the original definition, a 1-parameter family $M_{t}$ of hypersurfaces in $\tilde{M}^{n}(c)$ is called an isoparametric family if each $M_{t}$ is equal to a level set $V^{-1}(t)$ for some non-constant smooth real-valued function $V$ defined on a connected open subset of $\tilde{M}^{n}(c)$ such that the gradient and Laplacian of $V$ satisfy

$$
|\operatorname{grad} V|^{2}=T \circ V, \quad \triangle V=S \circ V,
$$

for some smooth functions $S$ and $T$. Thus, the two classical Beltrami differential parameters are both functions of $V$ itself, which leads to the name "isoparametric" for such a family of hypersurfaces. Such a function $V$ is called an isoparametric function. (See Thorbergsson [157, pp. 965-967] and Q.-M. Wang [163, 164] for more discussion of isoparametric functions.)

Let $f: M^{n-1} \rightarrow \tilde{M}^{n}(c)$ be an oriented hypersurface with field of unit normal vectors $\xi$. The parallel hypersurface to $f\left(M^{n-1}\right)$ at signed distance $t \in \mathbf{R}$ is the map $f_{t}: M^{n-1} \rightarrow \tilde{M}^{n}(c)$ such that for each $x \in M^{n-1}$, the point $f_{t}(x)$ is obtained by traveling a signed distance $t$ along the geodesic in $\tilde{M}^{n}(c)$ with initial point $f(x)$ and initial tangent vector $\xi(x)$. For $\tilde{M}^{n}(c)=\mathbf{R}^{n}$, the formula for $f_{t}$ is

$$
f_{t}(x)=f(x)+t \xi(x),
$$

and for $\tilde{M}^{n}(c)=S^{n}$, the formula for $f_{t}$ is

$$
f_{t}(x)=\cos t f(x)+\sin t \xi(x) .
$$

There is a similar formula in hyperbolic space (see, for example, [20]). 
Locally, for sufficiently small values of $t$, the map $f_{t}$ is also an immersed hypersurface. However, the map $f_{t}$ may develop singularities at the focal points of the original hypersurface $f\left(M^{n-1}\right)$. Specifically, a point $p=f_{t}(x)$ is called a focal point of multiplicity $m>0$ of $\left(f\left(M^{n-1}\right), x\right)$ if the differential $\left(f_{t}\right)_{*}$ has nullity $m$ at $x$. In the case $\tilde{M}^{n}(c)=\mathbf{R}^{n}$, respectively $S^{n}$, the point $p=f_{t}(x)$ is a focal point of multiplicity $m$ of $\left(f\left(M^{n-1}\right), x\right)$ if and only if $1 / t$, respectively $\cot t$, is a principal curvature of multiplicity $m$ of $f\left(M^{n-1}\right)$ at $x$ (see, for example, [83, pp. 32-38] or [38, pp. 243-247]).

Let $f: M^{n-1} \rightarrow \tilde{M}^{n}(c)$ be an oriented hypersurface with constant principal curvatures. One can show from the formulas for the principal curvatures of a parallel hypersurface (see, for example, [38, pp. 131-132]) that if $f$ has constant principal curvatures, then each $f_{t}$ that is an immersed hypersurface also has constant principal curvatures. However, since the principal curvatures of $f$ are constant on $M^{n-1}$, the focal points along the normal geodesic to $f\left(M^{n-1}\right)$ at $f(x)$ occur for the same values of $t$ independent of the choice of point $x \in M^{n-1}$. For example, for $\tilde{M}^{n}(c)=\mathbf{R}^{n}$, if $\mu$ is a non-zero constant principal curvature of multiplicity $m>0$ of $M^{n-1}$, the map $f_{1 / \mu}$ has constant rank $n-1-m$ on $M^{n-1}$, and the set $f_{1 / \mu}\left(M^{n-1}\right)$ is an $(n-1-m)$-dimensional submanifold of $\mathbf{R}^{n}$ called a focal submanifold of $f\left(M^{n-1}\right)$.

One can show (see, for example, [38, pp. 268-274]) that the level hypersurfaces of an isoparametric function $V$ form a family of parallel hypersurfaces (modulo reparametrization of the normal geodesics to take into account the possibility that $|\operatorname{grad} V|$ is not identically equal to one), and each of these level hypersurfaces has constant principal curvatures. Conversely, one can begin with a connected hypersurface $f: M^{n-1} \rightarrow \tilde{M}^{n}(c)$ having constant principal curvatures and construct an isoparametric function $V$ such that each parallel hypersurface $f_{t}$ of $f$ is contained in a level set of $V$. Therefore, one can define an isoparametric hypersurface to be a hypersurface with constant principal curvatures, and an isoparametric family of hypersurfaces can be characterized as a family of parallel hypersurfaces, each of which has constant principal curvatures. It is important that isoparametric hypersurfaces always come as a family of parallel hypersurfaces together with their focal submanifolds.

As noted in the introduction, an isoparametric hypersurface in $\mathbf{R}^{n}$ must be an open subset of a hyperplane, hypersphere or a spherical cylinder $S^{k} \times \mathbf{R}^{n-k-1}$ (Somigliana [134] for $n=3$, see also B. Segre [128] and Levi-Civita [75], and B. Segre [129] for arbitrary $n$ ). Shortly after the publication of the papers of Levi-Civita and Segre, Cartan $[8,9,10,11]$ undertook the study of isoparametric hypersurfaces in arbitrary real space-forms $\tilde{M}^{n}(c), c \in \mathbf{R}$, and we now describe his primary contributions.

Let $f: M^{n-1} \rightarrow \tilde{M}^{n}(c)$ be an isoparametric hypersurface with $g$ distinct principal curvatures $\mu_{1}, \ldots, \mu_{g}$, having respective multiplicities $m_{1}, \ldots, m_{g}$. If $g>1$, Cartan showed that for each $i$, $1 \leq i \leq g$,

$$
\sum_{j \neq i} m_{j} \frac{c+\mu_{i} \mu_{j}}{\mu_{i}-\mu_{j}}=0 .
$$

This important equation, known as Cartan's identity, is crucial in Cartan's work on isoparametric hypersurfaces. For example, using this identity, Cartan was able to classify isoparametric hypersurfaces in the cases $c \leq 0$ as follows. In the case $c=0$, if $g=1$, then $f$ is totally umbilic, and it is well known that $f\left(M^{n-1}\right)$ must be an open subset of a hyperplane or hypersphere. If $g \geq 2$, then by taking an appropriate choice of unit normal field $\xi$, one can assume that at least one of the principal curvatures is positive. If $\mu_{i}$ is the smallest positive principal curvature, then each term $\mu_{i} \mu_{j} /\left(\mu_{i}-\mu_{j}\right)$ in the sum in equation (4) is non-positive, and thus must equal zero. Therefore, there are at most two distinct principal curvatures, and if there are two, then one of them must be zero. Hence, $g=2$ and one can show $f\left(M^{n-1}\right)$ is an open subset of a spherical cylinder by standard methods in Euclidean hypersurface theory. 
In the case $c=-1$, if $g=1$, then $f$ is totally umbilic, and it is well known that $f\left(M^{n-1}\right)$ must be an open subset of a totally geodesic hyperplane, an equidistant hypersurface, a horosphere or a hypersphere in $H^{n}$ (see, for example, [135, p. 114]). If $g \geq 2$, then again one can arrange that at least one of the principal curvatures is positive. Then there must exist a positive principal curvature $\mu_{i}$ such that no principal curvature lies between $\mu_{i}$ and $1 / \mu_{i}$. (Here $\mu_{i}$ is either the largest principal curvature between 0 and 1 or the smallest principal curvature greater than or equal to one.) For this $\mu_{i}$, each term $\left(-1+\mu_{i} \mu_{j}\right) /\left(\mu_{i}-\mu_{j}\right)$ in the sum in equation (4) is negative unless $\mu_{j}=1 / \mu_{i}$. Thus, there are at most two distinct principal curvatures, and if there are two, then they are reciprocals of each other. Hence, $g=2$ and one can show that $f\left(M^{n-1}\right)$ is an open subset of a standard product $S^{k} \times H^{n-k-1}$ in hyperbolic space $H^{n}$ (see Ryan [126]).

In the sphere $S^{n}$, however, Cartan's identity does not lead to such strong restrictions on the number $g$ of distinct principal curvatures, and Cartan himself produced examples with $g=1,2,3$ or 4 distinct principal curvatures. Moreover, he classified isoparametric hypersurfaces $f: M^{n-1} \rightarrow S^{n}$ with $g \leq 3$ as follows. In the case $g=1$, the hypersurface $f$ is totally umbilic, and it is well known that $f\left(M^{n-1}\right)$ is an open subset of a great or small hypersphere in $S^{n}$ (see, for example, [135, p. 112]). If $g=2$, then $M$ must be a standard product of two spheres,

$$
S^{p}(r) \times S^{q}(s) \subset S^{n}(1) \subset \mathbf{R}^{p+1} \times \mathbf{R}^{q+1}=\mathbf{R}^{n+1}, \quad r^{2}+s^{2}=1,
$$

where $n=p+q+1$ (see, for example, [38, pp. 295-296]).

In the case of three distinct principal curvatures, Cartan [10] showed that all the principal curvatures must have the same multiplicity $m=1,2,4$ or 8 , and $f\left(M^{n-1}\right)$ must be a tube of constant radius over a standard embedding of a projective plane $\mathbf{F P}^{2}$ into $S^{3 m+1}$ (see, for example, [38, pp. 296-299]), where $\mathbf{F}$ is the division algebra $\mathbf{R}, \mathbf{C}, \mathbf{H}$ (quaternions), $\mathbf{O}$ (Cayley numbers), for $m=1,2,4,8$, respectively. (In the case $\mathbf{F}=\mathbf{R}$, a standard embedding is a Veronese surface in $S^{4}$.) Thus, up to congruence, there is only one such family of isoparametric hypersurfaces for each value of $m$. For each of these hypersurfaces, the focal set of $f\left(M^{n-1}\right)$ consists of two antipodal standard embeddings of $\mathbf{F P}^{2}$, and $f\left(M^{n-1}\right)$ is a tube of constant radius over each focal submanifold.

In the process of proving this theorem, Cartan showed that any isoparametric family with $g$ distinct principal curvatures of the same multiplicity can be defined by an equation of the form $F=\cos g t$ (restricted to $S^{n}$ ), where $F$ is a harmonic homogeneous polynomial of degree $g$ on $\mathbf{R}^{n+1}$ satisfying

$$
|\operatorname{grad} F|^{2}=g^{2} r^{2 g-2}
$$

where $r=|x|$ for $x \in \mathbf{R}^{n+1}$, and $\operatorname{grad} F$ is the gradient of $F$ in $\mathbf{R}^{n+1}$. This was a forerunner of Münzner's general result that every isoparametric hypersurface is algebraic, and its defining polynomial satisfies certain differential equations which generalize those that Cartan found in this special case.

In the case $g=4$, Cartan produced isoparametric hypersurfaces with four principal curvatures of multiplicity one in $S^{5}$ and four principal curvatures of multiplicity two in $S^{9}$. He noted all of his examples are homogeneous, each being an orbit of a point under an appropriate closed subgroup of $S O(n+1)$. Based on his results and the properties of his examples, Cartan asked the following three questions [10], all of which were answered in the 1970's.

1. For each positive integer $g$, does there exist an isoparametric family with $g$ distinct principal curvatures of the same multiplicity? 
2. Does there exist an isoparametric family of hypersurfaces with more than three distinct principal curvatures such that the principal curvatures do not all have the same multiplicity?

3. Does every isoparametric family of hypersurfaces admit a transitive group of isometries?

Despite the depth and beauty of Cartan's work, the subject of isoparametric hypersurfaces in $S^{n}$ was virtually ignored for thirty years until a revival of the subject in the early 1970's by several authors. Nomizu [102, 103] wrote two papers describing the highlights of Cartan's work. He also generalized Cartan's example with four principal curvatures of multiplicity one to produce examples with four principal curvatures having multiplicities $m_{1}=m_{2}=m$, and $m_{3}=m_{4}=1$, for any positive integer $m$. This answered Cartan's Question 2 in the affirmative. Nomizu also proved that every focal submanifold of every isoparametric hypersurface must be a minimal submanifold of $S^{n}$.

Takagi and Takahashi [143] gave a complete classification of all homogeneous isoparametric hypersurfaces in $S^{n}$, based on the work of Hsiang and Lawson [67]. Takagi and Takahashi showed that each homogeneous isoparametric hypersurface in $S^{n}$ is an orbit of the isotropy representation of a Riemannian symmetric space of rank 2, and they gave a complete list of examples [143, p. 480]. This list contains examples with six principal curvatures as well as those with $g=1,2,3,4$ principal curvatures, and in some cases with $g=4$, the principal curvatures do not all have the same multiplicity, so this also provided an affirmative answer to Cartan's Question 2.

At about the same time as the papers of Nomizu and Takagi-Takahashi, Münzner published two preprints that greatly extended Cartan's work and have served as the basis for much of the research in the field since that time. The preprints were eventually published as papers [93, 94] in $1980-1981$.

In the first paper [93] (see also Chapter 3 of [38]), Münzner began with a geometric study of the focal submanifolds of an isoparametric hypersurface $f: M^{n-1} \rightarrow S^{n}$ with $g$ distinct principal curvatures. Using the fact that each focal submanifold of $f$ is obtained as a parallel map $f_{t}$, where $\cot t$ is a principal curvature of $f\left(M^{n-1}\right)$, Münzner computed a formula for the shape operator of the focal submanifold $f_{t}\left(M^{n-1}\right)$ in terms of the shape operator of $f\left(M^{n-1}\right)$ itself. In particular, his calculation shows that the assumption that $f\left(M^{n-1}\right)$ has constant principal curvatures implies that the eigenvalues of the shape operator $A_{\eta}$ are the same for every unit normal $\eta$ at every point of the focal submanifold $f_{t}\left(M^{n-1}\right)$. Then by using a symmetry argument, he proved that if the principal curvatures of $f\left(M^{n-1}\right)$ are written as $\cot \theta_{k}, 0<\theta_{1}<\cdots<\theta_{g}<\pi$, with multiplicities $m_{k}$, then

$$
\theta_{k}=\theta_{1}+\frac{(k-1)}{g} \pi, \quad 1 \leq k \leq g,
$$

and the multiplicities satisfy $m_{k}=m_{k+2}$ (subscripts mod $g$ ). Thus, if $g$ is odd, all of the multiplicities must be equal, and if $g$ is even, there are at most two distinct multiplicities. Münzner's calculation shows further that the focal submanifolds must be minimal submanifolds of $S^{n}$, as Nomizu [102] had shown by a different proof, and that Cartan's identity is equivalent to the minimality of the focal submanifolds (see also [38, p. 251]).

If $\cot t$ is not a principal curvature of $f$, then the map $f_{t}$ in equation (3) is also an isoparametric hypersurface with $g$ distinct principal curvatures $\cot \left(\theta_{1}-t\right), \ldots, \cot \left(\theta_{g}-t\right)$. If $t=\theta_{k}$ $(\bmod \pi)$, then the map $f_{t}$ is constant along each leaf of the $m_{k}$-dimensional principal foliation $T_{k}$, and the image of $f_{t}$ is a smooth focal submanifold of $f$ of codimension $m_{k}+1$ in $S^{n}$. All of the hypersurfaces $f_{t}$ in a family of parallel isoparametric hypersurfaces have the same focal submanifolds. 
In a crucial step in the theory, Münzner then showed that $f\left(M^{n-1}\right)$ and its parallel hypersurfaces and focal submanifolds are each contained in a level set of a homogeneous polynomial $F$ of degree $g$ satisfying the following Cartan-Münzner differential equations on the Euclidean differential operators $\operatorname{grad} F$ and Laplacian $\triangle F$ on $\mathbf{R}^{n+1}$,

$$
\begin{aligned}
& |\operatorname{grad} F|^{2}=g^{2} r^{2 g-2}, \quad r=|x|, \\
& \triangle F=c r^{g-2}, \quad c=g^{2}\left(m_{2}-m_{1}\right) / 2,
\end{aligned}
$$

where $m_{1}, m_{2}$ are the two (possibly equal) multiplicities of the principal curvatures on $f\left(M^{n-1}\right)$. This generalized Cartan's polynomial in equation (6) for the case of $g$ principal curvatures with the same multiplicity.

Conversely, the level sets of the restriction $\left.F\right|_{S^{n}}$ of a function $F$ satisfying equation (8) constitute an isoparametric family of hypersurfaces and their focal submanifolds, and $F$ is called the Cartan-Münzner polynomial associated to this family. Furthermore, Münzner showed that the level sets of $F$ are connected, and thus any connected isoparametric hypersurface in $S^{n}$ lies in a unique compact, connected isoparametric hypersurface obtained by taking the whole level set.

The values of the restriction $\left.F\right|_{S^{n}}$ range between -1 and +1 . For $-1<t<1$, the level set $M_{t}=\left(\left.F\right|_{S^{n}}\right)^{-1}(t)$ is an isoparametric hypersurface, while $M_{+}=\left(\left.F\right|_{S^{n}}\right)^{-1}(1)$ and $M_{-}=$ $\left(\left.F\right|_{S^{n}}\right)^{-1}(-1)$ are focal submanifolds. Thus, there are exactly two focal submanifolds for the isoparametric family, regardless of the number $g$ of distinct principal curvatures. Each principal curvature $\cot \theta_{k}, 1 \leq k \leq g$, gives rise to two antipodal focal points corresponding to the values $t=\theta_{k}$ and $t=\theta_{k}+\pi$ in equation (7). The $2 g$ focal points are evenly spaced at intervals of length $\pi / g$ along a normal geodesic to the isoparametric family, and they lie alternately on the two focal submanifolds $M_{+}$and $M_{-}$, which have respective codimensions $m_{1}+1$ and $m_{2}+1$ in $S^{n}$.

Using this information, Münzner showed that each isoparametric hypersurface $M_{t}$ in the family separates the sphere $S^{n}$ into two connected components $D_{1}$ and $D_{2}$, such that $D_{1}$ is a disk bundle with fibers of dimension $m_{1}+1$ over $M_{+}$, and $D_{2}$ is a disk bundle with fibers of dimension $m_{2}+1$ over $M_{-}$, where $m_{1}$ and $m_{2}$ are the multiplicities of the principal curvatures that give rise to the focal submanifolds $M_{+}$and $M_{-}$, respectively.

This topological situation has been the basis for many important results in this field concerning the number $g$ of distinct principal curvatures and the multiplicities $m_{1}$ and $m_{2}$. In particular, in his second paper, Münzner [94] assumed that $M$ is a compact, connected embedded hypersurface that separates $S^{n}$ into two disk bundles $D_{1}$ and $D_{2}$ over compact manifolds with fibers of dimensions $m_{1}+1$ and $m_{2}+1$, respectively. From this hypothesis, Münzner proved that the dimension of the cohomological ring $H^{*}\left(M, \mathbf{Z}_{2}\right)$ must be $2 \alpha$, where $\alpha$ is one of the numbers $1,2,3,4$ or 6 . He then proved that if $M$ is a compact, connected isoparametric hypersurface with $g$ distinct principal curvatures, then $\operatorname{dim} H^{*}\left(M, \mathbf{Z}_{2}\right)=2 g$. Combining these two results, Münzner obtained his major theorem that the number $g$ of distinct principal curvatures of an isoparametric hypersurface in a sphere $S^{n}$ must be $1,2,3,4$ or 6 .

Münzner also obtained some restrictions on the possible values of the multiplicities $m_{1}$ and $m_{2}$. These restrictions have been improved by several authors also using topological arguments, as we will describe later in this section. In particular, in the case $g=4$, Abresch [1] showed that if $m_{1}=m_{2}=m$, then the only possible values for $m$ are 1 and 2 , the values in Cartan's examples. In fact, up to congruence, Cartan's examples are the only isoparametric hypersurfaces with four principal curvatures of the same multiplicity $m$. This was proven by Takagi [142] for $m=1$ and by Ozeki and Takeuchi [107] for $m=2$.

Münzner's result gave a negative answer to Cartan's Question 1, but it pointed towards an affirmative answer to Cartan's Question 3, since the possible values 1, 2, 3, 4 or 6 for 
the number $g$ of distinct principal curvatures of an isoparametric hypersurface agreed with the values of $g$ for the homogeneous isoparametric hypersurfaces on the list of Takagi and Takahashi. However, in an important 2-part paper, Ozeki and Takeuchi [107, 108] used representations of Clifford algebras to produce two infinite series of isoparametric families with four principal curvatures, most of which are necessarily inhomogeneous, because their multiplicities do not agree with the examples on the list of Takagi and Takahashi. These papers of Ozeki and Takeuchi were then vastly generalized by Ferus, Karcher and Münzner [58], who also used representations of Clifford algebras to produce a large and important class of isoparametric families with four principal curvatures which contains all known examples with $g=4$ with the exception of two homogeneous families. We will now briefly describe the construction of Ferus, Karcher and Münzner (see also, [26, pp. 95-112]).

For each integer $m \geq 0$, the Clifford algebra $C_{m}$ is the associative algebra over $\mathbf{R}$ that is generated by a unity 1 and the elements $e_{1}, \ldots, e_{m}$ subject only to the relations

$$
e_{i}^{2}=-1, \quad e_{i} e_{j}=-e_{j} e_{i}, \quad i \neq j, \quad 1 \leq i, j \leq m .
$$

One can show that the set

$$
\left\{1, e_{i_{1}} \cdots e_{i_{r}} \mid i_{1}<\cdots<i_{r}, 1 \leq r \leq m\right\}
$$

forms a basis for the underlying vector space $C_{m}$, and thus $\operatorname{dim} C_{m}=2^{m}$.

A representation of $C_{m}$ on $\mathbf{R}^{l}$ (of degree $l$ ) corresponds to a set of skew-symmetric matrices $E_{1}, \ldots, E_{m}$ in the orthogonal group $O(l)$ such that

$$
E_{i}^{2}=-I, \quad E_{i} E_{j}=-E_{j} E_{i}, \quad i \neq j, \quad 1 \leq i, j \leq m .
$$

Atiyah, Bott and Shapiro [3] determined all of the Clifford algebras according to the following table, and they showed that the Clifford algebra $C_{m-1}$ has an irreducible representation of degree $l$ if and only if $l=\delta(m)$ as in the table.

$\begin{array}{ccc}\underline{m} & \underline{C_{m-1}} & \underline{\delta(m)} \\ 1 & \mathbf{R} & 1 \\ 2 & \mathbf{C} & 2 \\ 3 & \mathbf{H} & 4 \\ 4 & \mathbf{H} \oplus \mathbf{H} & 4 \\ 5 & \mathbf{H}(2) & 8 \\ 6 & \mathbf{C}(4) & 8 \\ 7 & \mathbf{R}(8) & 8 \\ 8 & \mathbf{R}(8) \oplus \mathbf{R}(8) & 8 \\ k+8 & C_{k-1}(16) & 16 \delta(k)\end{array}$

Reducible representations of $C_{m-1}$ on $\mathbf{R}^{l}$ for $l=k \delta(m), k>1$, can be obtained by taking a direct sum of $k$ irreducible representations of $C_{m-1}$ on $\mathbf{R}^{\delta(m)}$.

Given a representation of $C_{m-1}$ on $\mathbf{R}^{l}$ corresponding to skew-symmetric matrices $E_{1}, \ldots$, $E_{m-1}$ satisfying equation (10), Ferus, Karcher and Münzner [58] construct an isoparametric family of hypersurfaces in the sphere $S^{2 l-1} \subset \mathbf{R}^{2 l}=\mathbf{R}^{l} \times \mathbf{R}^{l}$ with four principal curvatures such that one of the focal submanifolds is the manifold

$$
V_{2}\left(C_{m-1}\right)=\left\{(u, v) \in S^{2 l-1}|| u|=| v \mid=\frac{1}{\sqrt{2}}, u \cdot v=0, E_{i} u \cdot v=0,1 \leq i \leq m-1\right\} .
$$


This is the Clifford-Stiefel manifold of Clifford orthogonal 2-frames of length $1 / \sqrt{2}$ in $\mathbf{R}^{l}$ (see Pinkall and Thorbergsson [117]), where vectors $u$ and $v$ in $\mathbf{R}^{l}$ are said to be Clifford orthogonal if $u \cdot v=E_{1} u \cdot v=\cdots=E_{m-1} u \cdot v=0$, where $u \cdot v$ is the usual Euclidean inner product in $\mathbf{R}^{l}$.

Since $V_{2}\left(C_{m-1}\right)$ has codimension $m+1$ in $S^{2 l-1}$, one of the principal curvatures of an isoparametric hypersurface $M$ in this family has multiplicity $m_{1}=m$. The other multiplicity $m_{2}$ must satisfy the equation $2 m_{1}+2 m_{2}=\operatorname{dim} M=2 l-2$. Therefore $m_{2}=l-m-1$, where $l=k \delta(m)$ by the theorem of Atiyah, Bott and Shapiro.

The isoparametric hypersurfaces resulting from this are called isoparametric hypersurfaces of FKM-type. The following is a table of the multiplicities of the principal curvatures of the FKMhypersurfaces for multiplicities $\left(m_{1}, m_{2}\right)=(m, k \delta(m)-m-1)$ for small values of $m$. Of course, the multiplicity $m_{2}$ must be positive in order for this construction to lead to an isoparametric hypersurface with four principal curvatures. In the table below, the cases where $m_{2} \leq 0$ are denoted by a dash.

$\begin{array}{llllllllllllll}\delta(m) \mid & 1 & 2 & 4 & 4 & 8 & 8 & 8 & 8 & 16 & 32 & \ldots \\ \underline{k} & & & & & & & & & & & & & \\ 1 & - & - & - & - & (5,2) & (6,1) & - & - & (9,6) & (10,21) & \ldots \\ 2 & - & (2,1) & (3,4) & (4,3) & (5,10) & (6,9) & (7,8) & (8,7) & (9,22) & (10,53) & \ldots \\ 3 & (1,1) & (2,3) & (3,8) & (4,7) & (5,18) & (6,17) & (7,16) & (8,15) & (9,38) & (10,85) & \ldots \\ 4 & (1,2) & (2,5) & (3,12) & (4,11) & (5,26) & (6,25) & (7,24) & (8,23) & (9,54) & . & \ldots \\ 5 & (1,3) & (2,7) & (3,16) & (4,15) & (5,34) & (6,33) & (7,32) & (8,31) & . & . & \ldots \\ . & . & . & . & . & . & . & . & . & . & . & \ldots \\ . & . & . & . & . & . & . & . & . & . & . & \ldots \\ . & . & . & . & . & . & . & . & . & . & . & \ldots\end{array}$

Multiplicities of the principal curvatures of FKM-hypersurfaces

If $m \equiv 0(\bmod 4)$ and $l=k \delta(m)$, this construction yields $[k / 2]+1$ incongruent isoparametric families having the same multiplicities. Furthermore, the families with multiplicities $(2,1)$, $(6,1),(5,2)$ and one of the $(4,3)$-families are congruent to those with multiplicities $(1,2),(1,6)$, $(2,5)$ and $(3,4)$, respectively, and these are the only coincidences under congruence among the FKM-hypersurfaces [58].

Many of the FKM examples are necessarily inhomogeneous, because their multiplicities do not agree with the multiplicities of any hypersurface on the list of Takagi and Takahashi. However, Ferus, Karcher and Münzner also gave a geometric proof of the inhomogeneity of many of their examples through an examination of the second fundamental forms of the focal submanifolds. Later Q.-M. Wang [165] proved many results concerning the topology of the FKM examples and $\mathrm{Wu}$ [169] showed that there are only finitely many diffeomorphism classes of compact isoparametric hypersurfaces with four distinct principal curvatures.

All known examples of isoparametric hypersurfaces with four principal curvatures are of FKM-type with the exception of two homogeneous families, having multiplicities $(2,2)$ and $(4,5)$. Beginning with Münzner [93, 94], many mathematicians, including Abresch [1], Grove and Halperin [61], Tang [146] and Fang [53, 54], found restrictions on the multiplicities of the principal curvatures of an isoparametric hypersurface with four principal curvatures. This series of results culminated with the paper of Stolz [140], who proved that the multiplicities of an isoparametric hypersurface with four principal curvatures must be the same as those in the known examples of FKM-type or the two homogeneous exceptions. All of these papers are primarily topological in nature, based on Münzner's result that an isoparametric hypersurface separates $S^{n}$ into two disk bundles over the two focal submanifolds. In particular, the proof of Stolz is homotopy theoretic, and the main tools used are the Hopf invariant and the EHP-sequence. In fact, Stolz proved his result under the more general assumption that $M$ is a compact, connected proper Dupin (not 
necessarily isoparametric) hypersurface embedded in $S^{n}$. Note that Thorbergsson [155] had earlier shown that a compact, connected proper Dupin hypersurface $M \subset S^{n}$ also separates $S^{n}$ into two disk bundles over the first focal submanifolds on either side of $M$, as in the isoparametric case. This will be discussed in more detail in the next section.

Cecil, Chi and Jensen [29] then showed that if the multiplicities $\left(m_{1}, m_{2}\right)$ of an isoparametric hypersurface $M \subset S^{n}$ with four principal curvatures satisfy $m_{2} \geq 2 m_{1}-1$, then $M$ must be of FKM-type. Previously, Takagi [142] had shown that if one of the multiplicities $m_{1}=1$, then $M$ is homogeneous and of FKM-type. Ozeki and Takeuchi [108] next showed that if $m_{1}=2$, then $M$ is homogeneous and of FKM-type, except in the case of multiplicities $(2,2)$, in which case $M$ must be the homogeneous example of Cartan. Taken together with these results of Takagi and Ozeki-Takeuchi, the theorem of Cecil, Chi and Jensen classifies isoparametric hypersurfaces with four principal curvatures for all possible pairs of multiplicities except for four cases, the homogeneous pair $(4,5)$, and the FKM pairs $(3,4),(6,9)$ and $(7,8)$, for which the classification of isoparametric hypersurfaces remains an important open problem.

We now briefly outline the proof of this result by Cecil, Chi and Jensen. In Sections 8-9 of [29], Cecil, Chi and Jensen use Cartan's method of moving frames to find necessary and sufficient conditions (equations (8.1)-(8.4) of [29]) for the codimension $m_{1}+1$ focal submanifold $M_{+}$of an isoparametric hypersurface $M$ with four principal curvatures and multiplicities $\left(m_{1}, m_{2}\right)$ to be a Clifford-Stiefel manifold $V_{2}\left(C_{m_{1}-1}\right)$. (Later Chi [40] gave a different proof of the fact that equations (8.1)-(8.4) of [29] are necessary and sufficient to show that $M_{+}$is a Clifford-Stiefel manifold.)

These necessary and sufficient conditions involve the shape operators $A_{\eta}$ of $M_{+}$, where $\eta$ is a unit normal vector to $M_{+}$at a point $x \in M_{+}$. These shape operators are isospectral in that every $A_{\eta}$ at every point $x \in M_{+}$has the same eigenvalues $-1,0,1$, with respective multiplicities $m_{2}, m_{1}, m_{2}$. If $\eta$ is a unit normal vector to $M_{+}$at a point $x \in M_{+}$, then the point $\eta$ is also in $M_{+}$by Münzner's results, since it lies at a distance $\pi / 2$ along the normal geodesic to $M_{+}$beginning at the point $x$ in the direction $\eta$. The shape operators corresponding to an orthonormal basis of normal vectors to $M_{+}$at the point $x$ determine a family of $m_{1}+1$ homogeneous polynomials. Similarly, the shape operators corresponding to an orthonormal basis of normal vectors to $M_{+}$at the point $\eta$ determine a family of $m_{1}+1$ homogeneous polynomials. In Section 10 of [29], Cecil, Chi and Jensen show that these two families of polynomials have the same zero set in projective space by use of a formulation of the Cartan-Münzner polynomial due to Ozeki and Takeuchi [107]. Finally, in Sections 11-13 of [29], the authors employ techniques from algebraic geometry to show that the fact that these two sets of polynomials have the same zero set leads to a proof that the necessary and sufficient conditions for $M_{+}$to be a CliffordStiefel manifold are satisfied if $m_{2} \geq 2 m_{1}-1$. This completes the proof that $M$ is of FKM-type, since $M$ is a tube of constant radius over the Clifford-Stiefel manifold $M_{+}$.

After this, Immervoll [72] gave a different proof of the theorem of Cecil, Chi and Jensen using isoparametric triple systems. The use of triple systems to study isoparametric hypersurfaces was introduced in a series of papers in the 1980's by Dorfmeister and Neher [45, 46, 47, 48, 49, 50].

In the case of an isoparametric hypersurface with six principal curvatures, Münzner showed that all of the principal curvatures must have the same multiplicity $m$, and Abresch [1] showed that $m$ must equal 1 or 2 . By the classification of homogeneous isoparametric hypersurfaces due to Takagi and Takahashi [143], there is only one homogeneous family in each case up to congruence. In the case of multiplicity $m=1$, Dorfmeister and Neher [49] showed that an isoparametric hypersurface must be homogeneous, thereby completely classifying that case. The proof of Dorfmeister and Neher is quite algebraic in nature, and recently Miyaoka [89] has given a shorter, more geometric proof of this result.

Miyaoka [88] also gave a geometric description of the case $m=1$, showing that a homogeneous isoparametric hypersurface $M^{6}$ in $S^{7}$ can be obtained as the inverse image under the Hopf 
fibration $h: S^{7} \rightarrow S^{4}$ of an isoparametric hypersurface with three principal curvatures of multiplicity one in $S^{4}$. Miyaoka also showed that the two focal submanifolds of $M^{6}$ are not congruent, even though they are lifts under $h^{-1}$ of congruent Veronese surfaces in $S^{4}$. Thus, these focal submanifolds are two non-congruent minimal homogeneous embeddings of $\mathbf{R} \mathbf{P}^{2} \times S^{3}$ in $S^{7}$.

Peng and Hou [111] gave explicit forms for the Cartan-Münzner polynomials of degree six for the homogeneous isoparametric hypersurfaces with $g=6$, and Fang [55] proved several results concerning the topology of isoparametric and compact proper Dupin hypersurfaces with six principal curvatures.

The classification of isoparametric hypersurfaces with six principal curvatures of multiplicity $m=2$ is part of Problem 34 on Yau's [170] list of important open problems in geometry, and it remains an open problem. It has long been conjectured that the one homogeneous family in the case $g=6, m=2$, is the only isoparametric family in this case, but this conjecture has resisted proof for a long time. The approach that Miyaoka [89] used in the case $m=1$ shows promise of possibly leading to a proof of this conjecture, but so far a complete proof has not been published.

In the 1980's, the notion of an isoparametric hypersurface was extended to submanifolds of codimension greater than one in $S^{n}$ by several authors independently. (See Carter and West [17, 166], Harle [64], Strübing [141] and Terng [148].) An immersed submanifold $\phi: V \rightarrow \mathbf{R}^{n}$ (or $S^{n}$ ) is defined to be isoparametric if its normal bundle $N(V)$ is flat, and if for any locally defined normal field $\xi$ which is parallel with respect to the normal connection $\nabla^{\perp}$, the eigenvalues of the shape operator $A_{\xi}$ are constant. This theory was then developed extensively by many authors over the next decade, especially in the papers of Terng [148], Palais and Terng [109], and Hsiang, Palais and Terng [68] (see also the book [110]). Finally, Thorbergsson [156] used the theory of Tits buildings to show that all irreducible isoparametric submanifolds of codimension greater than one in $S^{n}$ are homogeneous, and therefore they are principal orbits of isotropy representations of symmetric spaces, also known as generalized flag manifolds or standard embeddings of $R$-spaces. (See Bott and Samelson [7], Takeuchi and Kobayashi [145], Dadok [42], Hahn [63] or the book by Berndt, Console and Olmos [6].) Later Olmos [105] and Heintze and Liu [65] gave alternate proofs of Thorbergsson's result.

Heintze, Olmos and Thorbergsson [66] defined a submanifold $\phi: V \rightarrow \mathbf{R}^{n}$ (or $S^{n}$ ) to have constant principal curvatures if for any smooth curve $\gamma$ on $V$ and any parallel normal vector field $\xi(t)$ along $\gamma$, the shape operator $A_{\xi(t)}$ has constant eigenvalues along $\gamma$. If the normal bundle $N(M)$ is flat, then having constant principal curvatures is equivalent to being isoparametric. They then showed that a submanifold with constant principal curvatures is either isoparametric or a focal submanifold of an isoparametric submanifold. The excellent survey article of Thorbergsson [157] gives a more detailed account of the theory of isoparametric submanifolds of codimension greater than one in $S^{n}$.

An immersion $\phi: V \rightarrow \mathbf{R}^{n}$ of a compact, connected manifold $V$ into $\mathbf{R}^{n}$ is said to be taut (see, for example, [13] or [38]) if every Morse function of the form $L_{p}: V \rightarrow \mathbf{R}$, where $L_{p}(x)=|p-\phi(x)|^{2}$, for $p \in \mathbf{R}^{n}$, has the minimum number of critical points required by the Morse inequalities using $\mathbf{Z}_{2}$-homology, i.e., it is a perfect Morse function. Tautness can also be studied for submanifolds of $S^{n}$ using spherical distance functions instead of Euclidean distance functions, and tautness is invariant under stereographic projection and its inverse map.

Isoparametric submanifolds (of any codimension) and their focal submanifolds are all taut submanifolds of $S^{n}$. This was proven by Cecil and Ryan [37] for hypersurfaces and by Hsiang, Palais and Terng [68] for isoparametric submanifolds of codimension greater than one in $S^{n}$, and this was an important fact in the general development of the theory.

Carter and West $[14,15,16,18]$, introduced the notion of totally focal submanifolds and studied its relationship to the isoparametric property. A submanifold $\phi: V \rightarrow \mathbf{R}^{n}$ is said to 
be totally focal if the critical points of every Euclidean distance function $L_{p}$ on $V$ are either all non-degenerate or all degenerate. An isoparametric submanifold in $S^{n}$ is totally focal, and the main result of [18] is that a totally focal submanifold must be isoparametric. However, Terng and Thorbergsson [154, p. 197] have noted that there is a gap in the proof of this assertion, specifically in the proof of Theorem 5.1 of [18].

$\mathrm{Wu}$ [168] and Zhao [171] generalized the theory of isoparametric submanifolds of codimension greater than one to submanifolds of hyperbolic space, and Verhóczki [159] developed a theory of isoparametric submanifolds for Riemannian manifolds which do not have constant curvature. West [167] and Mullen [92] formulated a theory of isoparametric systems on symmetric spaces, and Terng and Thorbergsson [153] studied compact isoparametric submanifolds of symmetric spaces using the related notion of equifocal submanifolds. Tang [147] then did a thorough study of the possible multiplicities of the focal points of equifocal hypersurfaces in symmetric spaces. In a generalization of Thorbergsson's result for submanifolds of $S^{n}$, Christ [41] proved that a complete connected irreducible equifocal submanifold of codimension greater than one in a simply connected compact symmetric space is homogeneous. Finally, a promising recent generalization of the theory of isoparametric submanifolds is the theory of singular Riemannian foliations admitting sections (see Alexandrino [2], Töben [158], and Lytchak and Thorbergsson $[80])$.

In a later paper, Terng and Thorbergsson [154] gave a definition of tautness for submanifolds of arbitrary complete Riemannian manifolds, and they discussed the notions of isoparametric, equifocal and Dupin submanifolds in that setting. In a related development, Carter and Şentürk [12] studied the space of immersions parallel to a given immersion whose normal bundle has trivial holonomy group.

Terng [151] considered isoparametric submanifolds in infinite-dimensional Hilbert spaces and generalized many results from the finite-dimensional case to that setting. Pinkall and Thorbergsson [118] then gave more examples of such submanifolds, and Heintze and Liu [65] generalized the finite-dimensional homogeneity result of Thorbergsson [156] to the infinite-dimensional case.

Nomizu [104] began the study of isoparametric hypersurfaces in pseudo-Riemannian space forms by proving a generalization of Cartan's identity for space-like hypersurfaces in a Lorentzian space form $\tilde{M}_{1}^{n}(c)$ of constant sectional curvature $c$. As a consequence of this identity, Nomizu showed that a space-like isoparametric hypersurface in $\tilde{M}_{1}^{n}(c)$ can have at most two distinct principal curvatures if $c \geq 0$. Recently, Li and Xie [77] have shown that this conclusion also holds for space-like isoparametric hypersurfaces in $\tilde{M}_{1}^{n}(c)$ for $c<0$. Magid [81] studied isoparametric hypersurfaces in Lorentz space whose shape operator is not diagonalizable, and Hahn [62] contributed an extensive study of isoparametric hypersurfaces in pseudoRiemannian space forms of arbitrary signatures. Recently Geatti and Gorodski [60] have extended this theory further by showing that a polar orthogonal representation of a connected real reductive algebraic group has the same closed orbits as the isotropy representation of a pseudo-Riemannian symmetric space. Working in a different direction, Niebergall and Ryan [97, 98, 99, 100] developed a theory of isoparametric and Dupin hypersurfaces in affine differential geometry.

There is also an extensive theory of real hypersurfaces with constant principal curvatures in complex space forms that is closely related to the theory of isoparametric hypersurfaces in spheres. See the survey articles of Niebergall and Ryan [101] and Berndt [5] for more detail.

In applications to Riemannian geometry, Solomon [131, 132, 133] found results concerning the spectrum of the Laplacian of isoparametric hypersurfaces in $S^{n}$ with three or four principal curvatures, and Eschenburg and Schroeder [52] studied the behavior of the Tits metric on isoparametric hypersurfaces. Finally, Ferapontov $[56,57]$ studied the relationship between isoparametric and Dupin hypersurfaces and Hamiltonian systems of hydrodynamic type and listed several open research problems in that context. 


\section{Dupin hypersurfaces}

As noted in the introduction, the theory of proper Dupin hypersurfaces is closely related to that of isoparametric hypersurfaces. In contrast to the situation for isoparametric hypersurfaces, however, there are both local and global aspects to the theory of proper Dupin hypersurfaces with quite different results concerning the number of distinct principal curvatures and their multiplicities, for example. In this section, we survey the primary results in this field. For the sake of completeness, we will begin with a formal definition of the Dupin and proper Dupin properties.

Via stereographic projection $\tau: S^{n}-\{P\} \rightarrow \mathbf{R}^{n}$ with pole $P \in S^{n}$ and its inverse map $\sigma$ from $\mathbf{R}^{n}$ into $S^{n}$, the theory of Dupin hypersurfaces is essentially the same for hypersurfaces in $\mathbf{R}^{n}$ or $S^{n}$ (see, for example, [38, pp. 132-151]), and we will use whichever ambient space is most convenient for the discussion at hand. Since we have been dealing with isoparametric hypersurfaces in spheres, we will formulate our definitions here in terms of hypersurfaces in $S^{n}$.

Let $f: M \rightarrow S^{n}$ be an immersed hypersurface, and let $\xi$ be a locally defined field of unit normals to $f(M)$. A curvature surface of $M$ is a smooth submanifold $S$ such that for each point $x \in S$, the tangent space $T_{x} S$ is equal to a principal space of the shape operator $A$ of $M$ at $x$. The hypersurface $M$ is said to be Dupin if:

(a) along each curvature surface, the corresponding principal curvature is constant.

The hypersurface $M$ is called proper Dupin if, in addition to Condition (a), the following condition is satisfied:

(b) the number $g$ of distinct principal curvatures is constant on $M$.

An obvious and important class of proper Dupin hypersurfaces are the isoparametric hypersurfaces in $S^{n}$, and those hypersurfaces in $\mathbf{R}^{n}$ obtained from isoparametric hypersurfaces in $S^{n}$ via stereographic projection. For example, the well-known ring cyclides of Dupin in $\mathbf{R}^{3}$ are obtained from a standard product torus $S^{1}(r) \times S^{1}(s) \subset S^{3}, r^{2}+s^{2}=1$, in this way. These examples will be discussed in more detail later in this section.

We begin by mentioning several well-known basic facts about Dupin hypersurfaces which are proven in Section 2.4 of [38], for example. As above, let $f: M \rightarrow S^{n}$ be an immersed hypersurface, and let $\xi$ be a locally defined field of unit normals to $f(M)$. First by the Codazzi equation, Condition (a) is automatically satisfied on a curvature surface $S$ of dimension greater than one. Second, Condition (b) is equivalent to requiring that each continuous principal curvature have constant multiplicity on $M$. Further, the number of distinct principal curvatures is locally constant on a dense open subset of any hypersurface in $S^{n}$ (see Singley [130]).

Next, if a continuous principal curvature function $\mu$ has constant multiplicity $m$ on a connected open subset $U \subset M$, then $\mu$ is a smooth function, and the distribution $T_{\mu}$ of principal vectors corresponding to $\mu$ is a smooth foliation whose leaves are the curvature surfaces corresponding to $\mu$. The principal curvature $\mu$ is constant along each of its curvature surfaces in $U$ if and only if these curvature surfaces are open subsets of $m$-dimensional great or small spheres in $S^{n}$. Suppose that $\mu=\cot \theta$, for $0<\theta<\pi$, where $\theta$ is a smooth function on $U$. The corresponding focal map $f_{\mu}$ which maps $x \in M$ to the focal point $f_{\mu}(x)$ is given by the formula,

$$
f_{\mu}(x)=\cos \theta(x) f(x)+\sin \theta(x) \xi(x) .
$$

The principal curvature $\mu$ also determines a second focal map, whose image is antipodal to the image of $f_{\mu}$, obtained by replacing $\theta$ by $\theta+\pi$ in equation (12). The principal curvature function $\mu$ is constant along each of its curvature surfaces in $U$ if and only if the focal map $f_{\mu}$ 
factors through an immersion of the $(n-1-m)$-dimensional space of leaves $U / T_{\mu}$ into $S^{n}$, and thus $f_{\mu}(U)$ is an $(n-1-m)$-dimensional submanifold of $S^{n}$.

The curvature sphere $K_{\mu}(x)$ corresponding to the principal curvature $\mu$ at a point $x \in U$ is the hypersphere in $S^{n}$ through $f(x)$ centered at the focal point $f_{\mu}(x)$. Thus, $K_{\mu}(x)$ is tangent to $f(M)$ at $f(x)$. The principal curvature $\mu$ is constant along each of its curvature surfaces if and only if the curvature sphere map $K_{\mu}$ is constant along each of these curvature surfaces.

Thus, on an open subset $U$ on which Condition (b) holds, Condition (a) is equivalent to requiring that each curvature surface in each principal foliation be an open subset of a metric sphere in $S^{n}$ of dimension equal to the multiplicity of the corresponding principal curvature. Condition (a) is also equivalent to the condition that along each curvature surface, the corresponding curvature sphere map is constant. Finally, on $U$, Condition (a) is equivalent to requiring that for each principal curvature $\mu$, the image of the focal map $f_{\mu}$ is a smooth submanifold of $S^{n}$ of codimension $m+1$, where $m$ is the multiplicity of $\mu$.

One consequence of these results is that like isoparametric hypersurfaces, proper Duper hypersurfaces are algebraic. For simplicity, we will formulate this result in terms of hypersurfaces of $\mathbf{R}^{n}$. The theorem states that a connected proper Dupin hypersurface $f: M \rightarrow \mathbf{R}^{n}$ must be contained in a connected component of an irreducible algebraic subset of $\mathbf{R}^{n}$ of dimension $n-1$. This result was widely thought to be true in the 1980's, and indeed Pinkall [113] sent the author a letter in 1984 that contained a sketch of a proof of this result. However, a proof was not published until recently by Cecil, Chi and Jensen [32], who used methods of real algebraic geometry to give a complete proof based on Pinkall's sketch. The proof makes use of the various principal foliations whose leaves are open subsets of spheres to construct an analytic algebraic parametrization of a neighborhood of $f(x)$ for each point $x \in M$. In contrast to the situation for isoparametric hypersurfaces, however, a connected proper Dupin hypersurface in $S^{n}$ does not necessarily lie in a compact connected proper Dupin hypersurface, as discussed later in this section. The algebraicity, and hence analyticity, of proper Dupin hypersurfaces was useful in clarifying certain fine points in the recent paper [32] of Cecil, Chi and Jensen on proper Dupin hypersurfaces with four principal curvatures.

The definition of Dupin can be extended to submanifolds of codimension greater than one as follows. Let $\phi: V \rightarrow \mathbf{R}^{n}$ (or $S^{n}$ ) be a submanifold of codimension greater than one, and let $B^{n-1}$ denote the unit normal bundle of $\phi(V)$. A curvature surface (see Reckziegel [121]) is a connected submanifold $S \subset V$ for which there is a parallel section $\eta: S \rightarrow B^{n-1}$ such that for each $x \in S$, the tangent space $T_{x} S$ is equal to some smooth eigenspace of the shape operator $A_{\eta}$. The submanifold $\phi(V)$ is said to be Dupin if along each curvature surface, the corresponding principal curvature of $A_{\eta}$ is constant, and a Dupin submanifold is proper Dupin if the number of distinct principal curvatures is constant on the unit normal bundle $B^{n-1}$. An isoparametric submanifold of codimension greater than one is always Dupin, but it may not be proper Dupin. (See [152, pp. 464-469] for more detail on this point.) Pinkall [114, p. 439] proved that every extrinsically symmetric submanifold of a real space form is Dupin. Takeuchi [144] then determined which of these are proper Dupin.

Pinkall $[114,115]$ situated the study of Dupin hypersurfaces in the context of Lie sphere geometry, and this approach has proven to be very useful in subsequent research in the field. In particular, Dupin submanifolds of codimension greater than one in $S^{n}$ can be studied naturally in this setting. Here we give a brief outline of this approach to Dupin hypersurfaces in $S^{n}$. (See Pinkall [115], Chern [39], Cecil and Chern [27], or the book [26] for more detail.)

A Lie sphere is an oriented hypersphere or a point sphere (zero radius) in $S^{n}$. The set of all Lie spheres in $S^{n}$ is in bijective correspondence with the set of points $[x]=\left[\left(x_{1}, \ldots, x_{n+3}\right)\right]$ (homogeneous coordinates) in real projective space $\mathbf{P}^{n+2}$ that lie on the quadric hypersurface $Q^{n+1}$ 
(the Lie quadric) determined by the equation $\langle x, x\rangle=0$, where

$$
\langle x, y\rangle=-x_{1} y_{1}+x_{2} y_{2}+\cdots+x_{n+2} y_{n+2}-x_{n+3} y_{n+3}
$$

is a bilinear form of signature $(n+1,2)$ on the indefinite inner product space $\mathbf{R}_{2}^{n+3}$. Here the sphere $S^{n}$ is identified with the unit sphere in $\mathbf{R}^{n+1} \subset \mathbf{R}_{2}^{n+3}$, where $\mathbf{R}^{n+1}$ is spanned by the standard basis vectors $\left\{e_{2}, \ldots, e_{n+2}\right\}$ in $\mathbf{R}_{2}^{n+3}$. Specifically, the oriented hypersphere with center $p \in S^{n}$ and signed radius $\rho$ (the sign denotes the orientation) corresponds to the point $[(\cos \rho, p, \sin \rho)]$ in $Q^{n+1}$. Point spheres $p$ in $S^{n}$ correspond to those points $[(1, p, 0)]$ in $Q^{n+1}$ with radius $\rho=0$.

The Lie quadric $Q^{n+1}$ contains projective lines but no linear subspaces of $\mathbf{P}^{n+2}$ of higher dimension. If $[x]$ and $[y]$ are points in $Q^{n+1}$, the line $[x, y]$ determined by $[x]$ and $[y]$ lies on $Q^{n+1}$ if and only if $\langle x, y\rangle=0$. This condition means that the hyperspheres in $S^{n}$ corresponding to $[x]$ and $[y]$ are in oriented contact, i.e., they are tangent and have the same orientation at the point of contact. For a point sphere and an oriented sphere, oriented contact means that the point lies on the sphere.

A Lie sphere transformation is a projective transformation of $\mathbf{P}^{n+2}$ that maps $Q^{n+1}$ to itself. In terms of the geometry of $S^{n}$, a Lie sphere transformation is a diffeomorphism on the space of Lie spheres that preserves oriented contact of spheres (see Pinkall [115, p. 431]), since it takes lines on $Q^{n+1}$ to lines on $Q^{n+1}$. The group of Lie sphere transformations is isomorphic to $O(n+1,2) /\{ \pm I\}$, where $O(n+1,2)$ is the orthogonal group for the metric in equation (13). A Lie sphere transformation that takes point spheres to point spheres is a Möbius transformation, i.e., it is induced by a conformal diffeomorphism of $S^{n}$, and the set of all Möbius transformations is a subgroup of the Lie sphere group. An example of a Lie sphere transformation that is not a Möbius transformation is a parallel transformations $P_{t}$, which fixes the center of each Lie sphere but adds $t$ to its signed radius. The group of Lie sphere transformations is generated by the union of the Möbius group and the group of all parallel transformations.

The manifold $\Lambda^{2 n-1}$ of projective lines on $Q^{n+1}$ has a contact structure, i.e., a globally defined 1-form $\omega$ such that $\omega \wedge d \omega^{n-1}$ never vanishes on $\Lambda^{2 n-1}$. The condition $\omega=0$ defines a codimension one distribution $D$ on $\Lambda^{2 n-1}$ which has integral submanifolds of dimension $n-1$ but none of higher dimension. A Legendre submanifold is one of these integral submanifolds of maximal dimension, i.e., an immersion $\lambda: M^{n-1} \rightarrow \Lambda^{2 n-1}$ such that $\lambda^{*} \omega=0$.

An oriented hypersurface $f: M^{n-1} \rightarrow S^{n}$ with field of unit normals $\xi: M^{n-1} \rightarrow S^{n}$ naturally induces a Legendre submanifold $\lambda=\left[k_{1}, k_{2}\right]$ (the line determined by the points $\left[k_{1}\right]$ and $\left[k_{2}\right]$ in $\left.Q^{n+1}\right)$, where

$$
k_{1}=(1, f, 0), \quad k_{2}=(0, \xi, 1) .
$$

For each $x \in M^{n-1},\left[k_{1}(x)\right]$ is the unique point sphere and $\left[k_{2}(x)\right]$ is the unique great sphere in the parabolic pencil of spheres in $S^{n}$ corresponding to the points on the line $\lambda(x)$. Similarly, an immersed submanifold $\phi: V \rightarrow S^{n}$ of codimension greater than one induces a Legendre submanifold whose domain is the bundle $B^{n-1}$ of unit normal vectors to $\phi(V)$. In each case, $\lambda$ is called the Legendre lift of the submanifold in $S^{n}$. Note that the space of Legendre submanifolds is larger than the space of Legendre lifts of submanifolds of $S^{n}$, since the point sphere map of an arbitrary Legendre submanifold may not have constant rank as a map into $S^{n}$.

A Lie sphere transformation $\beta$ maps lines on $Q^{n+1}$ to lines on $Q^{n+1}$, so it naturally induces a map $\tilde{\beta}$ from $\Lambda^{2 n-1}$ to itself. If $\lambda$ is a Legendre submanifold, then $\tilde{\beta} \lambda$ is also a Legendre submanifold, which is denoted $\beta \lambda$ for short. These two Legendre submanifolds are said to be Lie equivalent. We will also say that two submanifolds of $S^{n}$ are Lie equivalent, if their corresponding Legendre lifts are Lie equivalent. If $\beta$ is a Möbius transformation, then the two Legendre submanifolds are said to be Möbius equivalent. Finally, if $\beta$ is the parallel transformation $P_{t}$ 
and $\lambda$ is the Legendre submanifold induced by an oriented hypersurface $f: M \rightarrow S^{n}$, then $P_{t} \lambda$ is the Legendre lift of the parallel hypersurface $f_{-t}$ at oriented distance $-t$ from $f$ (see, for example, [26, p. 67]).

To define the Dupin property for a Legendre submanifold $\lambda: M^{n-1} \rightarrow \Lambda^{2 n-1}$, one replaces the principal curvature function on $M^{n-1}$ in Conditions (a) and (b) above with the corresponding curvature sphere map $K: M^{n-1} \rightarrow Q^{n+1}$, which is naturally defined in this setting. One can easily show that a Lie sphere transformation $\beta$ maps curvatures spheres of $\lambda$ to curvature spheres of $\beta \lambda$, and that Conditions (a) and (b) are preserved by $\beta$ (see [26, pp. 67-70]). Thus, both the Dupin and proper Dupin properties are invariant under Lie sphere transformations.

Pinkall [115] introduced four standard constructions for obtaining a proper Dupin hypersurface in $\mathbf{R}^{n+m}$ from a proper Dupin hypersurface in $\mathbf{R}^{n}$. We first describe Pinkall's constructions in the case $m=1$.

Start with a Dupin hypersurface $W^{n-1}$ in $\mathbf{R}^{n}$ and then consider $\mathbf{R}^{n}$ as the linear subspace $\mathbf{R}^{n} \times\{0\}$ in $\mathbf{R}^{n+1}$. The following constructions yield a Dupin hypersurface $M^{n}$ in $\mathbf{R}^{n+1}$.

(1) Let $M^{n}$ be the cylinder $W^{n-1} \times \mathbf{R}$ in $\mathbf{R}^{n+1}$.

(2) Let $M^{n}$ be the hypersurface in $\mathbf{R}^{n+1}$ obtained by rotating $W^{n-1}$ around an axis $\mathbf{R}^{n-1} \subset$ $\mathbf{R}^{n}$.

(3) Project $W^{n-1}$ stereographically onto a hypersurface $V^{n-1} \subset S^{n} \subset \mathbf{R}^{n+1}$. Let $M^{n}$ be the cone over $V^{n-1}$ in $\mathbf{R}^{n+1}$.

(4) Let $M^{n}$ be a tube in $\mathbf{R}^{n+1}$ around $W^{n-1}$.

Even though Pinkall gave these four constructions, he noted that the tube construction and the cone construction are Lie equivalent [115, p. 438], and therefore in the context of Lie sphere geometry, it is sufficient to deal only with the tube, cylinder and surface of revolution constructions.

In general, these constructions introduce a new principal curvature of multiplicity one which is easily seen to be constant along its lines of curvature. The other principal curvatures are determined by the principal curvatures of $W^{n-1}$, and the Dupin property is preserved for these principal curvatures (see [22] or [26, pp. 127-141] for a full discussion of these constructions in the setting of Lie sphere geometry). Thus, if $W^{n-1}$ is a proper Dupin hypersurface in $\mathbf{R}^{n}$ with $g$ distinct principal curvatures, then in general, $M^{n}$ is a proper Dupin hypersurface in $\mathbf{R}^{n+1}$ with $g+1$ distinct principal curvatures. However, this is not always the case, as we will explain below. Pinkall also pointed out that these constructions can easily be generalized to produce a new principal curvature of multiplicity $m>1$ by considering the constructions in $\mathbf{R}^{n} \times \mathbf{R}^{m}$ instead of $\mathbf{R}^{n} \times \mathbf{R}$.

Let us now examine the possible problems encountered in trying to obtain a proper Dupin hypersurface by each of the three constructions. In all cases, suppose that $W^{n-1}$ is a proper Dupin hypersurface in $\mathbf{R}^{n}$ with $g$ distinct principal curvatures.

In the cylinder construction, the new principal curvature of $M^{n}$ is identically zero while the other principal curvatures of $M^{n}$ are equal to those of $W^{n-1}$. Thus if one of the principal curvatures $\kappa$ of $W^{n-1}$ is zero at some points but not identically zero, then the number of distinct principal curvatures is not constant on $M^{n}$, and $M^{n}$ is Dupin but not proper Dupin.

If $M^{n}$ is a tube in $\mathbf{R}^{n+1}$ of radius $\epsilon$ over $W^{n-1}$, then there are exactly two distinct principal curvatures at the points in the set $W^{n-1} \times\{ \pm \epsilon\}$ in $M^{n}$, regardless of the number of distinct principal curvatures on $W^{n-1}$. Thus, $M^{n}$ is not a proper Dupin hypersurface unless the original hypersurface $W^{n-1}$ is totally umbilic, i.e., it has only one distinct principal curvature at each point.

For the surface of revolution construction, if the focal point corresponding to a principal curvature $\kappa$ at a point $x$ of the profile submanifold $W^{n-1}$ lies on the axis of revolution $\mathbf{R}^{n-1}$, 
then the principal curvature of $M^{n}$ at $x$ determined by $\kappa$ is equal to the new principal curvature of $M^{n}$ resulting from the surface of revolution construction. Thus, if the focal point of $W^{n-1}$ corresponding to $\kappa$ lies on the axis of revolution for some but not all points of $W^{n-1}$, then $M^{n}$ is not proper Dupin.

Another problem with these constructions is that they may not yield an immersed hypersurface in $\mathbf{R}^{n+1}$. In the tube construction, if the radius of the tube is the reciprocal of one of the principal curvatures of $W^{n-1}$ at some point, then the constructed object has a singularity. For the surface of revolution construction, a singularity occurs if the profile submanifold $W^{n-1}$ intersects the axis of revolution. These problems can be resolved by working in the context of Lie sphere geometry (see [26, pp. 127-141]).

As noted above, these constructions can be generalized to the setting of Lie sphere geometry by considering Legendre lifts of hypersurfaces in Euclidean space. In that context, a proper Dupin submanifold $\lambda: M^{n-1} \rightarrow \Lambda^{2 n-1}$ is said to be reducible if it is is locally Lie equivalent to the Legendre lift of a hypersurface in $\mathbf{R}^{n}$ obtained by one of Pinkall's constructions.

Pinkall [115] found a useful characterization of reducibility in the context of Lie sphere geometry when he proved that a proper Dupin submanifold $\lambda: M^{n-1} \rightarrow \Lambda^{2 n-1}$ is reducible if and only if the image of one its curvature sphere maps $K$ lies in a linear subspace of codimension two in $\mathbf{P}^{n+2}$. Much of the recent research in the field has focused on the classification of irreducible proper Dupin hypersurfaces.

The following example (see [26, pp. 132-133] for more detail) shows that Pinkall's constructions can produce a proper Dupin submanifold with the same number of distinct curvature spheres as the original proper Dupin submanifold, rather than increasing the number of distinct curvature spheres by one.

Let $V^{2}$ be a Veronese surface embedded in the unit sphere $S^{4} \subset \mathbf{R}^{5}$. Let $N^{3}$ be a tube of radius $\varepsilon$, for $0<\varepsilon<\pi / 3$, over $V^{2}$ in $S^{4}$. Then $N^{3}$ is an isoparametric hypersurface $S^{4}$ with three distinct principal curvatures, and $N^{3}$ is an irreducible proper Dupin hypersurface, since Cecil, Chi and Jensen [30] proved that a compact, connected proper Dupin hypersurface $M^{n-1} \subset S^{n}$ with $g \geq 3$ principal curvatures is irreducible. $N^{3}$ is not the result of the tube construction, because the Veronese surface is substantial (does not lie in a hyperplane) in $\mathbf{R}^{5}$. Now embed $\mathbf{R}^{5}$ as a hyperplane through the origin in $\mathbf{R}^{6}$, and let $e_{6}$ be a unit normal vector to $\mathbf{R}^{5}$ in $\mathbf{R}^{6}$. The Veronese surface $V^{2}$ is a codimension three submanifold of the unit sphere $S^{5} \subset \mathbf{R}^{6}$. A tube $M^{4}$ over $V^{2}$ in $S^{5}$ is a reducible Dupin hypersurface, because the family of curvatures spheres coming from the tube construction lies in a linear space of codimension two in $\mathbf{P}^{7}$, since these curvature spheres all have the same radius and their centers all lie in the space $\mathbf{R}^{5}$. This tube $M^{4}$ is Dupin but not proper Dupin. At points of the tube $M^{4}$ coming from points of the form $\left(x, \pm e_{6}\right)$ in the unit normal bundle $B^{4}$ of $V^{2}$ in $S^{5}$, there are two distinct principal curvatures, both of multiplicity two. At the other points of $M^{4}$, there are three distinct principal curvatures, one of multiplicity two, and the other two of multiplicity one. Thus, the open dense subset $U$ of $M^{4}$ on which there are three principal curvatures is a reducible proper Dupin hypersurface, but $M^{4}$ itself is not proper Dupin. The number $g=3$ of distinct principal curvatures (or curvature spheres) on $U$ is the same as the number of distinct curvature spheres of the Legendre lift $\lambda$ of the original submanifold $V^{2} \subset S^{4}$, since the point sphere map of $\lambda$ is a curvature sphere map, due to the fact that $V^{2}$ has codimension greater than one in $S^{4}$. The other two curvature spheres of $\lambda$ are determined by the two principal curvatures of the surface $V^{2}$.

This example is important for illustrating some of the subtleties involved in studying the concept of reducibility. For example, Dajczer, Florit and Tojeiro [43] studied reducibility in the context of Euclidean submanifold theory. They formulated a concept of weak reducibility for proper Dupin submanifolds that have a flat normal bundle. In particular, they defined a proper Dupin hypersurface $f: M^{n-1} \rightarrow \mathbf{R}^{n}$ (or $S^{n}$ ) to be weakly reducible if, for some principal curvature $\kappa_{i}$ with corresponding principal space $T_{i}$, the orthogonal complement $T_{i}^{\perp}$ is integrable. 
Dajczer, Florit and Tojeiro showed that if a proper Dupin hypersurface $f: M^{n-1} \rightarrow \mathbf{R}^{n}$ is Lie equivalent to a proper Dupin hypersurface with $g+1$ distinct principal curvatures that is obtained from a proper Dupin hypersurface with $g$ distinct principal curvatures by one of the standard constructions, then $f$ is weakly reducible. Thus, reducible implies weakly reducible for such hypersurfaces. However, one can show that the open set $U$ with three principal curvatures in the example above is reducible but not weakly reducible, because none of the orthogonal complements of the principal spaces is integrable. Note that $U$ is not constructed from a proper Dupin submanifold with two curvature spheres, but rather one from one with three curvature spheres.

Cecil and Jensen [33, 34] defined a proper Dupin hypersurface $M^{n-1}$ in $\mathbf{R}^{n}$ to be locally irreducible if $M^{n-1}$ does not contain a reducible open subset. A locally irreducible proper Dupin hypersurface is obviously irreducible, and using the analyticity of proper Dupin hypersurfaces, Cecil, Chi and Jensen [30] proved conversely that an irreducible proper Dupin hypersurface is locally irreducible. Thus, the two concepts are equivalent.

Using his constructions listed above, Pinkall [115] (see also [26, p. 126]) demonstrated that proper Dupin hypersurfaces are plentiful. Specifically, he showed that given positive integers $m_{1}, \ldots, m_{g}$ with $m_{1}+\cdots+m_{g}=n-1$, there exists a proper Dupin hypersurface $M^{n-1}$ in $\mathbf{R}^{n}$ with $g$ distinct principal curvatures having respective multiplicities $m_{1}, \ldots, m_{g}$. The proper Dupin hypersurfaces that Pinkall constructs in his proof are all reducible, and in general, they cannot be completed to a compact proper Dupin hypersurface, because of the difficulties discussed above. In fact, compact proper Dupin hypersurfaces are much more rare, as we will describe below.

Recall from Section 2 that an immersion $\phi: V \rightarrow \mathbf{R}^{n}$ of a compact, connected manifold $V$ into $\mathbf{R}^{n}$ is taut if every non-degenerate Euclidean distance function $L_{p}, p \in \mathbf{R}^{n}$, has the minimum number of critical points on $V$ required by the Morse inequalities using $\mathbf{Z}_{2}$-homology. Tautness can also be studied for submanifolds of $S^{n}$ using spherical distance functions.

A taut submanifold of $S^{n}$ (or $\mathbf{R}^{n}$ ) must be Dupin, although not necessarily proper Dupin. This was first shown for compact surfaces in $\mathbf{R}^{3}$ by Banchoff [4] (see also [21] for the noncompact case), then by Miyaoka [85] for hypersurfaces, and independently by Pinkall [116] for submanifolds of arbitrary codimension.

Conversely, Thorbergsson [155] proved that a compact, connected proper Dupin hypersurface $M^{n-1} \subset S^{n}$ (or $\mathbf{R}^{n}$ ) with $g$ distinct principal curvatures is taut. Thorbergsson used the principal foliations on $M^{n-1}$ to construct concrete $\mathbf{Z}_{2}$-cycles in $M^{n-1}$ which show that all critical points of non-degenerate distance functions $L_{p}$ are of linking type (see Morse and Cairns [91, p. 258]). Therefore such distance functions have the minimum number $\beta\left(M^{n-1}\right)=2 g$ critical points, where $\beta\left(M^{n-1}\right)$ is the sum of the $\mathbf{Z}_{2}$-Betti numbers of $M^{n-1}$. Pinkall [116] later extended Thorbergsson's result to compact submanifolds of higher codimension for which the number of distinct principal curvatures is constant on the unit normal bundle.

Note that unlike the case for isoparametric hypersurfaces, the focal submanifolds of a compact, connected proper Dupin hypersurface $M^{n-1}$ need not be taut. For example, for a ring cyclide of Dupin $M^{2} \subset \mathbf{R}^{3}$ obtained by inverting a torus of revolution in a sphere, one of the focal submanifolds is an ellipse, which is not a taut embedding of $S^{1}$.

Ozawa [106] proved that if $V$ is a taut compact submanifold of $\mathbf{R}^{n}$ (or $S^{n}$ ), then every connected component $S$ of a critical set of a distance function $L_{p}$ on $V$ is a smooth submanifold of $V$, which is non-degenerate as a critical submanifold in the sense of Morse-Bott critical point theory (see [7]), and $S$ itself is taut in $\mathbf{R}^{n}$. Using Ozawa's result, one can prove (see [25, p. 154]) that if $M^{n-1} \subset \mathbf{R}^{n}$ is a taut compact, connected hypersurface, then given any principal space $T_{\mu}$ at any point $x \in M^{n-1}$, there is a curvature surface $S$ through $x$ whose tangent space is equal to $T_{\mu}$, and $\mu$ is constant along $S$. Thus $M^{n-1}$ is Dupin in this strong sense of having a curvature surface tangent to every principal space (this is not assumed as part of Condition (a) in the definition of a Dupin hypersurface). An important open ques- 
tion is whether the converse of this theorem is true. That is, if $M^{n-1} \subset \mathbf{R}^{n}$ is a compact, connected non-proper Dupin hypersurface with the property that given any principal space $T_{\mu}$ at any point $x \in M^{n-1}$, there is a curvature surface $S$ through $x$ whose tangent space is equal to $T_{\mu}$, must $M^{n-1}$ be taut? Thorbergsson's proof that a compact proper Dupin hypersurface must be taut relies on the fact that all the curvature surfaces are spheres. In the non-proper Dupin case, Ozawa's work implies that there are some curvature surfaces that are not spheres.

Terng defined a Dupin submanifold $V$ of arbitrary codimension to have constant multiplicities if the multiplicities of the principal curvatures of any parallel normal field $\xi(t)$ along any piecewise smooth curve on $V$ are constant. Terng [150], [152, p. 467] then proved that a compact, connected Dupin submanifold with constant multiplicities is taut.

Using tautness, Thorbergsson [155] proved that a compact, connected proper Dupin hypersurface $M^{n-1} \subset S^{n}$ separates $S^{n}$ into two disk bundles over the first focal submanifolds on either side of $M^{n-1}$, as in the case of isoparametric hypersurfaces. Thus, Münzner's restriction that the number of distinct principal curvatures must be 1,2, 3, 4 or 6 holds for compact, connected proper Dupin hypersurfaces $M^{n-1} \subset S^{n}$ also. Furthermore, the restrictions on the possible multiplicities of the principal curvatures due to Stolz [140] in the case $g=4$ and Grove and Halperin [61] in the case $g=6$ still hold. These results have led to a pursuit of classification results for compact proper Dupin hypersurfaces based on the number $g$ of distinct principal curvatures.

In the case $g=1$, it is well known that a compact, connected proper Dupin hypersurface $M^{n-1} \subset S^{n}$ must be a great or small hypersphere. In the case $g=2$, Cecil and Ryan [35] (see also [36] or [38, p. 168]) showed that $M^{n-1}$ must be Möbius equivalent to an isoparametric hypersurface, i.e., a standard product of spheres $S^{k}(r) \times S^{n-k-1}(s) \subset S^{n}, r^{2}+s^{2}=1$. In the case $g=3$, Miyaoka [84] proved that a compact, connected proper Dupin hypersurface $M^{n-1} \subset S^{n}$ must be Lie equivalent to an isoparametric hypersurface, although not necessarily Möbius equivalent.

These results together with Thorbergsson's restriction on the number $g$ of distinct principal curvatures led to the widely held conjecture [38, p. 184] that every compact, connected proper Dupin hypersurface $M^{n-1} \subset S^{n}$ is Lie equivalent to an isoparametric hypersurface in a sphere. However, in 1988 Pinkall and Thorbergsson [117], and Miyaoka and Ozawa [90] gave two different methods for producing counterexamples to this conjecture with four principal curvatures. The method of Miyaoka and Ozawa also yields counterexamples to the conjecture in the case of six principal curvatures.

Both of these constructions involve a consideration of the Lie curvatures of a proper Dupin hypersurface. If $M^{n-1} \subset S^{n}$ is a proper Dupin hypersurface with $g \geq 4$ distinct principal curvatures, then the cross-ratios of the principal curvatures taken four at a time are called the Lie curvatures of $M^{n-1}$. These Lie curvatures were first studied by Miyaoka [86] who proved that they are invariant under Lie sphere transformations. This is actually quite easy to see in the context of projective geometry, since each Lie curvature is the cross-ratio of four points (corresponding to curvature spheres) on a projective line in $\mathbf{P}^{n+2}$. A Lie sphere transformation $\beta$ is a projective transformation and it maps curvature spheres of a Legendre submanifold $\lambda$ to curvature spheres of the Legendre submanifold $\beta \lambda$, so it preserves the cross-ratios of the curvature spheres, and therefore it preserves the cross-ratios of the principal curvatures (see also [26, p. 75]).

The counterexamples of Pinkall and Thorbergsson [117] (see also [26, pp. 112-117]) to the conjecture are obtained by modifying the isoparametric hypersurfaces of FKM-type discussed in the previous section. Recall that the Clifford-Stiefel manifold $V_{2}\left(C_{m-1}\right)$ is a submanifold of $S^{2 l-1} \subset \mathbf{R}^{2 l}=\mathbf{R}^{l} \times \mathbf{R}^{l}$ of codimension $m+1$. Given positive real numbers $\alpha$ and $\beta$ with $\alpha^{2}+\beta^{2}=1$, where $\alpha \neq 1 / \sqrt{2}, \beta \neq 1 / \sqrt{2}$, Pinkall and Thorbergsson define a linear map 


$$
\begin{gathered}
T_{\alpha, \beta}: \mathbf{R}^{2 l} \rightarrow \mathbf{R}^{2 l}, \text { by } T_{\alpha, \beta}(u, v)=\sqrt{2}(\alpha u, \beta v) \text {. Then for }(u, v) \in V_{2}\left(C_{m-1}\right), \text { we have } \\
\left|T_{\alpha, \beta}(u, v)\right|^{2}=2\left(\alpha^{2}(u \cdot u)+\beta^{2}(v \cdot v)\right)=2\left(\frac{\alpha^{2}}{2}+\frac{\beta^{2}}{2}\right)=1,
\end{gathered}
$$

and thus the image $T_{\alpha, \beta} V_{2}\left(C_{m-1}\right)$ is a submanifold of $S^{2 l-1}$ of codimension $m+1$ also. Pinkall and Thorbergsson prove that a tube over $T_{\alpha, \beta} V_{2}\left(C_{m-1}\right)$ in $S^{2 l-1}$ is a compact, connected proper Dupin hypersurface with four principal curvatures that does not have constant Lie curvature, and therefore it is not Lie equivalent to an isoparametric hypersurface.

The construction of counterexamples to the conjecture due to Miyaoka and Ozawa [90] (see also [26, pp. 117-123]) is based on the Hopf fibration $h: S^{7} \rightarrow S^{4}$. Miyaoka and Ozawa first show that if $M$ is a taut compact submanifold of $S^{4}$, then $h^{-1}(M)$ is a taut compact submanifold of $S^{7}$. Using this, they next show that if $M$ is a proper Dupin hypersurface in $S^{4}$ with $g$ distinct principal curvatures, then $h^{-1}(M)$ is a proper Dupin hypersurface in $S^{7}$ with $2 g$ principal curvatures. To complete the argument, they show that if a hypersurface $M \subset S^{4}$ is proper Dupin but not isoparametric, then the Lie curvatures of $h^{-1}(M)$ are not constant, and therefore $h^{-1}(M)$ is not Lie equivalent to an isoparametric hypersurface in $S^{7}$. For $g=2$ or 3 , respectively, this gives a compact proper Dupin hypersurface with 4 or 6 principal curvatures, respectively, in $S^{7}$, which is not Lie equivalent to an isoparametric hypersurface.

These counterexamples are both based on the fact that the constructed proper Dupin hypersurface does not have constant Lie curvatures. This leads to a revision of the conjecture [31, p. 52] which states that every compact, connected proper Dupin hypersurface $M^{n-1} \subset S^{n}$ with $g=4$ or 6 principal curvatures and constant Lie curvatures is Lie equivalent to an isoparametric hypersurface in a sphere. This revised conjecture is still an important open problem, although it has has been shown to be true in some cases, as we now describe.

Miyaoka $[86,87]$ began by showing that if some additional assumptions are made regarding the intersections of the leaves of the various principal foliations, then this revised conjecture is true in both cases $g=4$ and $g=6$. Thus far, however, it has not been shown that Miyaoka's additional assumptions are satisfied in general. In the case $g=6$, this work of Miyaoka is the only known progress towards proving the revised conjecture.

If $M^{n-1} \subset S^{n}$ is a compact, connected proper Dupin hypersurface with four principal curvatures having multiplicities $m_{1}, m_{2}, m_{3}, m_{4}$, then the multiplicities must satisfy $m_{1}=m_{2}$, $m_{3}=m_{4}$, when the principal curvatures are appropriately ordered, by the work of Thorbergsson [155] and Münzner [93, 94]. Cecil, Chi and Jensen [30] have recently shown that if $M^{n-1} \subset S^{n}$ is a compact, connected proper Dupin hypersurface with four principal curvatures having multiplicities $m_{1}=m_{2} \geq 1, m_{3}=m_{4}=1$, and constant Lie curvature, then $M^{n-1}$ is Lie equivalent to an isoparametric hypersurface. This result actually follows from a local classification of irreducible proper Dupin hypersurfaces with four principal curvatures [30], which we will discuss below. Thus, the full revised conjecture in the case $g=4$ would be proven if one could remove the assumption $m_{3}=m_{4}=1$, but so far this has not been done.

In his early work on the subject, Pinkall [112, 114, 115], proved some important local classification results concerning proper Dupin hypersurfaces, and this approach has been extended to more general settings by using the notion of irreducibility. Although it has not been shown that irreducibility places any restriction on the number $g$ of distinct principal curvatures other than $g \geq 3$, most of the known results for irreducible proper Dupin hypersurfaces have been obtained in the cases $g=3$ or 4 .

As with compact proper Dupin hypersursurfaces, we will discuss the known local classification results for proper Dupin hypersurfaces of $S^{n}$ based on the number $g$ of distinct principal curvatures. It is well known, that a connected proper Dupin hypersurface in $S^{n}$ with $g=1$ (totally umbilic) distinct principal curvature must be an open subset of a hypersphere. 
In the case $g=2$, Pinkall [114] obtained a complete local classification which we now describe in detail. As a generalization of the well-known cyclides of Dupin in $\mathbf{R}^{3}$, Pinkall defined a cyclide of Dupin of characteristic $(p, q)$ to be a proper Dupin hypersurface in $S^{n}$ (or $\mathbf{R}^{n}$ ) with two distinct principal curvatures of respective multiplicities $p$ and $q$. An example of a cyclide of Dupin of characteristic $(p, q)$ is the standard product of spheres, $S^{p}(r) \times S^{q}(s) \subset S^{n}, r^{2}+s^{2}=1$, where $n=p+q+1$, as in equation (5). This is an isoparametric hypersurface in $S^{n}$ with two principal curvatures, as discussed earlier. If one varies the value of $r$ in equation (5) between 0 and 1, one obtains an isoparametric family of hypersurfaces with two principal curvatures. The hypersurfaces in this family are Lie equivalent by parallel transformation, but they are not Möbius equivalent for different values of $r$.

Pinkall proved that every connected cyclide of Dupin is contained in a unique compact, connected cyclide, and any two cyclides of the same characteristic $(p, q)$ are locally Lie equivalent. Thus, every cyclide of Dupin of characteristic $(p, q)$ is locally Lie equivalent to a standard product of spheres, as in equation (5).

Using Pinkall's Lie geometric classification one can derive the following Möbius geometric classification of the cyclides of Dupin [24] (see also [26, p. 151]). Every connected cyclide $M^{n-1}$ of characteristic $(p, q)$ in $\mathbf{R}^{n}$ is Möbius equivalent to an open subset of a hypersurface of revolution obtained by revolving a $q$-sphere $S^{q} \subset \mathbf{R}^{q+1} \subset \mathbf{R}^{n}$ about an axis of revolution $\mathbf{R}^{q} \subset \mathbf{R}^{q+1}$ or a $p$-sphere $S^{p} \subset \mathbf{R}^{p+1} \subset \mathbf{R}^{n}$ about an axis $\mathbf{R}^{p} \subset \mathbf{R}^{p+1}$. Further, two such hypersurfaces of revolution are Möbius equivalent if and only if they have the same value of $\rho=r / a$, where $r$ is the radius of the profile sphere $S^{q}$ and $a>0$ is the distance from the center of $S^{q}$ to the axis of revolution. In this theorem, the profile sphere is allowed to intersect the axis of revolution, which results in Euclidean singularities. However, in the context of Lie sphere geometry, the corresponding Legendre map is an immersion.

The classical cyclides of Dupin in $\mathbf{R}^{3}$ were first studied by Dupin [51] in 1822 and later by many prominent nineteenth century mathematicians, including Liouville [79], Cayley [19], and Maxwell [82], whose paper contains stereoscopic figures of the various types of cyclides. (See Lilienthal [78] for an account of the nineteenth century work on the cyclides.) More recent descriptions of the classical cyclides are contained in the books of Fladt and Baur [59, pp. 354379], Cecil and Ryan [38, pp. 151-166], and [26, pp. 148-159].

The classical cyclides are the only surfaces in $\mathbf{R}^{3}$ with two principal curvatures at each point such that all lines of curvature in both families are circles or straight lines. This is just the proper Dupin condition, of course. Using exterior differential systems, Ivey [73] showed that any surface in $\mathbf{R}^{3}$ containing two orthogonal families of circles is a cyclide of Dupin. The classical cyclides have also been used in computer aided geometric design of surfaces. See, for example, the papers of Degen [44], Pratt [119, 120], Srinivas and Dutta [136, 137, 138, 139], and Schrott and Odehnal [127].

Pinkall began the study of proper Dupin hypersurfaces with three distinct principal curvatures in his dissertation [112], published as a paper [115] (see also [28] or [26, pp. 168-190]). Pinkall found a complete local classification up to Lie equivalence for Dupin hypersurfaces with three principal curvatures in $\mathbf{R}^{4}$. He proved that any two irreducible proper Dupin hypersurfaces with $g=3$ in $\mathbf{R}^{4}$ are locally Lie equivalent, each being Lie equivalent to an open subset of Cartan's isoparametric hypersurface in $S^{4}$. For reducible proper Dupin hypersurfaces with $g=3$ in $\mathbf{R}^{4}$, Pinkall showed that there is a 1-parameter family of Lie equivalence classes.

Niebergall [95] next proved that every connected proper Dupin hypersurface in $\mathbf{R}^{5}$ with three principal curvatures is reducible. Then Cecil and Jensen [33] proved that if $M^{n-1}$ is an irreducible, connected proper Dupin hypersurface in $S^{n}$ with three distinct principal curvatures of multiplicities $m_{1}, m_{2}, m_{3}$, then $m_{1}=m_{2}=m_{3}=m$, and $M^{n-1}$ is Lie equivalent to an isoparametric hypersurface in $S^{n}$. It then follows from Cartan's classification of isoparametric hypersurfaces with $g=3$ mentioned in Section 2 that $m=1,2,4$ or 8 . Note that in the original 
paper [33], this result was proven under the assumption that $M^{n-1}$ is locally irreducible. However, as noted above, local irreducibility has now been shown to be equivalent to irreducibility.

The proof of this result of Cecil and Jensen is accomplished by using Cartan's method of moving frames in the context of Lie sphere geometry. A key tool in this context is the result (see [23] or [26, p. 77]) that a Legendre submanifold $\lambda: M^{n-1} \rightarrow \Lambda^{2 n-1}$ with $g$ distinct curvature spheres $K_{1}, \ldots, K_{g}$ at each point is Lie equivalent to the Legendre lift of an isoparametric hypersurface in $S^{n}$ if and only if there exist $g$ points $P_{1}, \ldots, P_{g}$ on a timelike line in $P^{n+2}$ such that $\left\langle K_{i}, P_{i}\right\rangle=0$, for $1 \leq i \leq g$.

An open problem is the classification of reducible Dupin hypersurfaces with three principal curvatures up to Lie equivalence. As noted above, Pinkall [115] found such a classification in the case of $M^{3} \subset \mathbf{R}^{4}$. It may be possible to generalize Pinkall's result to higher dimensions using the approach of [33].

Cecil, Chi and Jensen [30] proved that a compact, connected proper Dupin hypersurface $M^{n-1} \subset S^{n}$ with $g \geq 3$ principal curvatures is irreducible. Thus, the examples above of Pinkall and Thorbergsson [117] and Miyaoka and Ozawa [90] of compact proper Dupin hypersurfaces with $g=4$ and non-constant Lie curvature are irreducible proper Dupin hypersurfaces with $g=4$ that are not Lie equivalent to an isoparametric hypersurface. However, it is still possible that every irreducible Dupin hypersurface with $g=4$ and constant Lie curvature is Lie equivalent to an isoparametric hypersurface, and this has been shown under additional assumptions, as we now describe.

The first result in this direction is due to Niebergall [96] who showed that a connected irreducible proper Dupin hypersurface $M^{4}$ in $S^{5}$ with four principal curvatures and constant Lie curvature is Lie equivalent to an isoparametric hypersurface under an additional assumption that in an appropriate moving frame, the covariant derivatives of certain naturally defined functions are zero. Cecil and Jensen [34] then showed that Niebergall's additional assumptions are unnecessary, because these functions are always constant in the appropriate Lie frame. Thus, they proved that every connected irreducible proper Dupin hypersurface $M^{4}$ in $S^{5}$ with four principal curvatures and constant Lie curvature is Lie equivalent to an isoparametric hypersurface.

This result was then generalized to higher dimensions by Cecil, Chi and Jensen [30] who proved that if $M^{n-1}$ is an irreducible, connected proper Dupin hypersurface in $S^{n}$ with four principal curvatures having multiplicities $m_{1}=m_{2} \geq 1$ and $m_{3}=m_{4}=1$ and constant Lie curvature -1 , then $M^{n-1}$ is Lie equivalent to an isoparametric hypersurface. Note that Münzner [93, 94] had shown earlier that if $M^{n-1} \subset S^{n}$ is an isoparametric hypersurface with four principal curvatures, then the multiplicities of the principal curvatures must satisfy $m_{1}=m_{2}$ and $m_{3}=m_{4}$, and the Lie curvature must equal -1 , if the principal curvatures are appropriately ordered.

These results lead to the following local conjecture [31, p. 53]: if $M^{n-1}$ is an irreducible, connected, proper Dupin hypersurface in $S^{n}$ with four principal curvatures having multiplicities $m_{1}, m_{2}, m_{3}, m_{4}$, and constant Lie curvature $c$, then $m_{1}=m_{2}, m_{3}=m_{4}, c=-1$, and $M^{n-1}$ is Lie equivalent to an isoparametric hypersurface. Note that it has not yet been shown that irreducibility implies that $m_{1}=m_{2}$ and $m_{3}=m_{4}$, nor that $c=-1$.

We remark that the hypothesis of irreducibility is definitely needed in the conjecture because one can construct reducible non-compact proper Dupin hypersurfaces with $g=4$ and constant Lie curvature $c$, for every negative value of $c$, if the principal curvatures are appropriately ordered (see [23] or [26, pp. 80-82]). This construction yields examples where the multiplicities satisfy $m_{1}=m_{2}$ and $m_{3}=m_{4}$, and also examples where the multiplicities do not satisfy this restriction. These examples are all obtained as open subsets of a tube in $S^{n}$ over an isoparametric hypersurface with three principal curvatures $V^{k-1} \subset S^{k} \subset S^{n}$, and they cannot be completed to be compact proper Dupin hypersurfaces. They are also reducible as Dupin hypersurfaces. 
Using a different approach based on the theory of higher-dimensional Laplace invariants [74], Riveros and Tenenblat $[123,124]$ gave a local classification of proper Dupin hypersurfaces $M^{4}$ in $\mathbf{R}^{5}$ with four distinct principal curvatures which are parametrized by lines of curvature.

C.-P. Wang [160, 162] studied the Möbius geometry of submanifolds in $S^{n}$ in a series of papers. Using Cartan's method of moving frames, Wang found a complete set of Möbius invariants for surfaces in $\mathbf{R}^{3}$ without umbilic points [160] and for hypersurfaces in $\mathbf{R}^{4}$ with three principal curvatures at each point [161]. Then in [162], Wang defined a Möbius invariant metric $g$ and second fundamental form $B$ for submanifolds in $S^{n}$. Wang then proved that for hypersurfaces in $S^{n}$ with $n \geq 4$, the pair $(g, B)$ forms a complete Möbius invariant system which determines the hypersurface up to Möbius transformations.

In a related result, Riveros, Rodrigues and Tenenblat [122] proved that a proper Dupin hypersurface $M^{n} \subset \mathbf{R}^{n+1}, n \geq 4$, with $n$ distinct principal curvatures and constant Möbius curvatures cannot be parametrized by lines of curvature. They also showed that up to Möbius transformations, there is a unique proper Dupin hypersurface $M^{3} \subset \mathbf{R}^{4}$ with three principal curvatures and constant Möbius curvature that is parametrized by lines of curvature. This $M^{3}$ is a cone in $\mathbf{R}^{4}$ over a standard flat torus in the unit sphere $S^{3} \subset \mathbf{R}^{4}$.

In [76], H. Li, Lui, Wang and Zhao introduced the concept of a Möbius isoparametric hypersurface in a sphere $S^{n}$. They showed that a (Euclidean) isoparametric hypersurface is automatically Möbius isoparametric, whereas a Möbius isoparametric hypersurface must be proper Dupin. Later Rodrigues and Tenenblat [125] showed that if $M \subset S^{n}$ is a hypersurface with a constant number $g$ of distinct principal curvatures at each point, where $g \geq 3$, then $M$ is Möbius isoparametric if and only if $M$ is Dupin with constant Möbius curvatures.

Recently significant progress has been made in the classification of Möbius isoparametric hypersurfaces. First, H. Li, Lui, Wang and Zhao [76] showed that a connected Möbius isoparametric hypersurface in $S^{n}$ with two distinct principal curvatures is Möbius equivalent to an open subset of one of the following three types of hypersurfaces in $S^{n}$ :

(i) a standard product of spheres $S^{k}(r) \times S^{n-k-1}(s) \subset S^{n}, r^{2}+s^{2}=1$,

(ii) the image under inverse stereographic projection from $\mathbf{R}^{n} \rightarrow S^{n}-\{P\}$ of a standard cylinder $S^{k}(1) \times \mathbf{R}^{n-k-1} \subset \mathbf{R}^{n}$,

(iii) the image under hyperbolic stereographic projection from $H^{n} \rightarrow S^{n}$ of a standard product $S^{k}(r) \times H^{n-k-1}\left(\sqrt{1+r^{2}}\right) \subset H^{n}$.

Later $\mathrm{Hu}$ and $\mathrm{H}$. Li [70] classified Möbius isoparametric hypersurfaces in $S^{4}$, and $\mathrm{Hu}, \mathrm{H}$. Li and Wang [71] classified Möbius isoparametric hypersurfaces in $S^{5}$. Then Hu and D. Li [69] studied Möbius isoparametric hypersurfaces with three distinct principal curvatures in $S^{n}$ and found a complete classification of such hypersurfaces in $S^{6}$.

\section{Acknowledgements}

This material is based upon work supported by the National Science Foundation under Grant No. 0405529. The author is grateful for several helpful comments in the reports of the referees.

\section{References}

[1] Abresch U., Isoparametric hypersurfaces with four or six distinct principal curvatures, Math. Ann. 264 (1983), 283-302.

[2] Alexandrino M., Singular Riemannian foliations with sections, Illinois J. Math. 48 (2004), 1163-1182, math.DG/0311454.

[3] Atiyah M.F., Bott R., Shapiro A., Clifford modules, Topology 3 (1964), suppl. 1, 3-38. 
[4] Banchoff T., The spherical two-piece property and tight surfaces in spheres, J. Differential Geom. 4 (1970), 193-205.

[5] Berndt J., Real hypersurfaces with constant principal curvatures in complex space forms, in Proceedings of the Tenth International Workshop on Differential Geometry, Kyungpook Nat. Univ., Taegu, 2006, 1-12.

[6] Berndt J., Console S., Olmos C., Submanifolds and holonomy, Chapman and Hall/CRC Research Notes in Mathematics, Vol. 434, Chapman and Hall/CRC, Boca Raton, Florida, 2003.

[7] Bott R., Samelson H., Applications of the theory of Morse to symmetric spaces, Amer. J. Math. 80 (1958), 964-1029.

[8] Cartan É., Familles de surfaces isoparamétriques dans les espaces à courbure constante, Annali di Mat. 17 (1938), 177-191 (see also in Oeuvres Complètes, Partie III, Vol. 2, 1431-1445).

[9] Cartan É., Sur des familles remarquables d'hypersurfaces isoparamétriques dans les espaces sphériques, Math. Z. 45 (1939), 335-367 (see also in Oeuvres Complètes, Partie III, Vol. 2, 1447-1479).

[10] Cartan É., Sur quelque familles remarquables d'hypersurfaces, in C.R. Congrès Math. Liège, 1939, 30-41 (see also in Oeuvres Complètes, Partie III, Vol. 2, 1481-1492).

[11] Cartan É., Sur des familles d'hypersurfaces isoparamétriques des espaces sphériques à 5 et à 9 dimensions, Revista Univ. Tucuman, Serie A, 1 (1940), 5-22 (see also in Oeuvres Complètes, Partie III, Vol. 2, 15131530).

[12] Carter S., Şentürk Z., The space of immersions parallel to a given immersion, J. London Math. Soc. (2) 50 (1994), 404-416.

[13] Carter S., West A., Tight and taut immersions, Proc. London Math. Soc. (3) 25 (1972), 701-720.

[14] Carter S., West A., Totally focal embeddings, J. Differential Geom. 13 (1978), 251-261.

[15] Carter S., West A., Totally focal embeddings: special cases, J. Differential Geom. 16 (1981), 685-697.

[16] Carter S., West A., A characterisation of isoparametric hypersurfaces in spheres, J. London Math. Soc. (2) 26 (1982), 183-192.

[17] Carter S., West A., Isoparametric systems and transnormality, Proc. London Math. Soc. (3) 51 (1985), $520-542$.

[18] Carter S., West A., Isoparametric and totally focal submanifolds, Proc. London Math. Soc. (3) 60 (1990), 609-624.

[19] Cayley A., On the cyclide, Quart. J. of Pure and Appl. Math. 12 (1873), $148-165$ (see also in Collected Mathematical Papers, Vol. 9, Cambridge U. Press, 1896, 64-78).

[20] Cecil T., A characterization of metric spheres in hyperbolic space by Morse theory, Tôhoku Math. J. (2) $\mathbf{2 6}$ (1974), 341-351.

[21] Cecil T., Taut immersions of non-compact surfaces into a Euclidean 3-space, J. Differential Geom. 11 (1976), 451-459.

[22] Cecil T., Reducible Dupin submanifolds, Geom. Dedicata 32 (1989), 281-300.

[23] Cecil T., On the Lie curvatures of Dupin hypersurfaces, Kodai Math. J. 13 (1990), 143-153.

[24] Cecil T., Lie sphere geometry and Dupin submanifolds, in Geometry and Topology of Submanifolds III (Leeds, 1990), Editors L. Verstraelen and A. West, World Scientific, River Edge, NJ, 1991, 90-107.

[25] Cecil T., Taut and Dupin submanifolds, in Tight and Taut Submanifolds (Berkeley, CA, 1994), Editors T. Cecil and S.-S. Chern, Math. Sci. Res. Inst. Publ., Vol. 32, Cambridge Univ. Press, Cambridge, 1997, $135-180$.

[26] Cecil T., Lie sphere geometry, with applications to submanifolds, 2nd ed., Universitext, Springer, New York, 2008.

[27] Cecil T., Chern S.-S., Tautness and Lie sphere geometry, Math. Ann. 278 (1987), 381-399.

[28] Cecil T., Chern S.-S., Dupin submanifolds in Lie sphere geometry, in Differential Geometry and Topology (Tianjin, 1986-87), Editors B. Jiang et al., Lecture Notes in Math., Vol. 1369, Springer, Berlin - New York, 1989, 1-48.

[29] Cecil T., Chi Q.-S., Jensen G., Isoparametric hypersurfaces with four principal curvatures, Ann. of Math. (2) 166 (2007), 1-76.

[30] Cecil T., Chi Q.-S., Jensen G., Dupin hypersurfaces with four principal curvatures. II, Geom. Dedicata 128 (2007), 55-95. 
[31] Cecil T., Chi Q.-S., Jensen G., Classifications of Dupin hypersurfaces, in Pure and Applied Differential Geometry, PADGE 2007, Editors F. Dillen and I. van de Woestyne, Shaker Verlag, Aachen, 2007, 48-56.

[32] Cecil T., Chi Q.-S., Jensen G., On Kuiper's question whether taut submanifolds are algebraic, Pacific J. Math. 234 (2008), 229-247.

[33] Cecil T., Jensen G., Dupin hypersurfaces with three principal curvatures, Invent. Math. 132 (1998), 121-178.

[34] Cecil T., Jensen G., Dupin hypersurfaces with four principal curvatures, Geom. Dedicata 79 (2000), 1-49.

[35] Cecil T., Ryan P., Focal sets, taut embeddings and the cyclides of Dupin, Math. Ann. 236 (1978), 177-190.

[36] Cecil T., Ryan P., Conformal geometry and the cyclides of Dupin, Canad. J. Math. 32 (1980), 767-782.

[37] Cecil T., Ryan P., Tight spherical embeddings, in Global Differential Geometry and Analysis (Berlin 1979), Editors D. Ferus, W. Kühnel, U. Simon and B. Wegner, Lecture Notes in Math., Vol. 838, Springer, Berlin New York, 1981, 94-104.

[38] Cecil T., Ryan P., Tight and taut immersions of manifolds, Research Notes in Math., Vol. 107, Pitman, London, 1985.

[39] Chern S.-S., An introduction to Dupin submanifolds, in Differential Geometry: A Symposium in Honour of Manfredo do Carmo (Rio de Janeiro, 1988), Editors H.B. Lawson and K. Tenenblat, Pitman Monographs Surveys Pure Appl. Math., Vol. 52, Longman Sci. Tech., Harlow, 1991, 95-102.

[40] Chi Q.-S., Isoparametric hypersurfaces with four principal curvatures revisited, in Nagoya Math. J., to appear, arXiv:0803.1284.

[41] Christ U., Homogeneity of equifocal submanifolds, J. Differential Geom. 62 (2002), 1-15.

[42] Dadok J., Polar coordinates induced by actions of compact Lie groups, Trans. Amer. Math. Soc. 288 (1985), $125-137$.

[43] Dajczer M., Florit L., Tojeiro R., Reducibility of Dupin submanifolds, Illinois J. Math. 49 (2005), 759-791.

[44] Degen W., Generalized cyclides for use in CAGD, in Computer-Aided Surface Geometry and Design: the Mathematics of Surfaces IV (Bath, 1990), Editor A. Bowyer, Inst. Math. Appl. Conf. New Ser., Vol. 48, Oxford Univ. Press, New York, 1994, 349-363.

[45] Dorfmeister J., Neher E., An algebraic approach to isoparametric hypersurfaces. I, Tôhoku Math. J. (2) 35 (1983), 187-224.

Dorfmeister J., Neher E., An algebraic approach to isoparametric hypersurfaces. II, Tôhoku Math. J. (2) 35 (1983), 225-247.

[46] Dorfmeister J., Neher E., Isoparametric triple systems of algebra type, Osaka J. Math. 20 (1983), 145-175.

[47] Dorfmeister J., Neher E., Isoparametric triple systems of FKM-type. I, Abh. Math. Sem. Hamburg 53 (1983), $191-216$.

[48] Dorfmeister J., Neher E., Isoparametric triple systems of FKM-type. II, Manuscripta Math. 43 (1983), $13-44$.

[49] Dorfmeister J., Neher E., Isoparametric hypersurfaces, case $g=6, m=1$, Comm. Algebra 13 (1985), $2299-2368$

[50] Dorfmeister J., Neher E., Isoparametric triple systems with special Z-structure, Algebras Groups Geom. 7 (1990), 21-94.

[51] Dupin C., Applications de géométrie et de méchanique, Bachelier, Paris, 1822.

[52] Eschenburg J.-H., Schroeder V., Tits distance of Hadamard manifolds and isoparametric hypersurfaces, Geom. Dedicata 40 (1991), 97-101.

[53] Fang F., Multiplicities of principal curvatures of isoparametric hypersurfaces, Preprint, Max Planck Institut für Mathematik, Bonn, 1996.

[54] Fang F., On the topology of isoparametric hypersurfaces with four distinct principal curvatures, Proc. Amer. Math. Soc. 127 (1999), 259-264.

[55] Fang F., Topology of Dupin hypersurfaces with six principal curvatures, Math. Z. 231 (1999), 533-555.

[56] Ferapontov E.V., Dupin hypersurfaces and integrable hamiltonian systems of hydrodynamic type, which do not possess Riemann invariants, Differential Geom. Appl. 5 (1995), 121-152.

[57] Ferapontov E.V., Isoparametric hypersurfaces in spheres, integrable nondiagonalizable systems of hydrodynamic type, and $N$-wave systems, Differential Geom. Appl. 5 (1995), 335-369. 
[58] Ferus D., Karcher H., Münzner H.-F., Cliffordalgebren und neue isoparametrische Hyperflächen, Math. Z. 177 (1981), 479-502.

[59] Fladt K., Baur A., Analytische Geometrie spezieller Flächen und Raumkurven, Friedr. Vieweg and Sohn, Braunschweig, 1975.

[60] Geatti L., Gorodski C., Polar orthogonal representations of real reductive algebraic groups, J. Algebra, to appear, arXiv:0801.0574.

[61] Grove K., Halperin S., Dupin hypersurfaces, group actions, and the double mapping cylinder, J. Differential Geom. 26 (1987), 429-459.

[62] Hahn J., Isoparametric hypersurfaces in the pseudo-Riemannian space forms, Math. Z. 187 (1984), $195-208$.

[63] Hahn J., Isotropy representations of semisimple symmetric spaces and homogeneous hypersurfaces, J. Math. Soc. Japan 40 (1988), 271-288.

[64] Harle C., Isoparametric families of submanifolds, Bol. Soc. Brasil Mat. 13 (1982), no. 2, 35-48.

[65] Heintze E., Liu X., Homogeneity of infinite dimensional isoparametric submanifolds, Ann. of Math. (2) 149 (1999), 149-181, math.DG/9901150.

[66] Heintze E., Olmos C., Thorbergsson G., Submanifolds with constant principal curvatures and normal holonomy groups, Internat. J. Math. 2 (1991), 167-175.

[67] Hsiang W.-Y., Lawson H. B., Jr., Minimal submanifolds of low cohomogeneity, J. Differential Geom. 5 (1971), 1-38.

[68] Hsiang W.-Y., Palais R., Terng C.-L., The topology of isoparametric submanifolds, J. Differential Geom. 27 (1988), 423-460.

[69] Hu Z., Li D., Möbius isoparametric hypersurfaces with three distinct principal curvatures, Pacific J. Math. 232 (2007), 289-311.

[70] Hu Z., Li H.-Z., Classification of Möbius isoparametric hypersurfaces in $S^{4}$, Nagoya Math. J. 179 (2005), 147-162.

[71] Hu Z., Li H.-Z., Wang C.-P., Classification of Möbius isoparametric hypersurfaces in $S^{5}$, Monatsh. Math. 151 (2007), 201-222.

[72] Immervoll S., On the classification of isoparametric hypersurfaces with four distinct principal curvatures in spheres, Ann. Math., to appear.

[73] Ivey T., Surfaces with orthogonal families of circles, Proc. Amer. Math. Soc. 123 (1995), 865-872.

[74] Kamran N., Tenenblat K., Laplace transformation in higher dimensions, Duke Math. J. 84 (1996), $237-266$.

[75] Levi-Civita T., Famiglie di superficie isoparametrische nell'ordinario spacio euclideo, Atti. Accad. naz. Lincei. Rend. Cl. Sci. Fis. Mat. Natur. 26 (1937), 355-362.

[76] Li H.-Z., Liu H.-L., Wang C.-P., Zhao G.-S., Möbius isoparametric hypersurfaces in $S^{n+1}$ with two distinct principal curvatures, Acta Math. Sin. (Engl. Ser.) 18 (2002), 437-446.

[77] Li Z.-Q., Xie X.-H., Space-like isoparametric hypersurfaces in Lorentzian space forms, J. Nanchang Univ. Natur. Sci. Ed. 28 (2004), 113-117 (English transl: Front. Math. China 1 (2006), 130-137).

[78] Lilienthal R., Besondere Flächen, in Encyklopädie der Math. Wissenschaften, Vol. III, Teubner, Leipzig, 1902-1927, 269-354.

[79] Liouville J., Note au sujet de l'article précedént, J. de Math. Pure et Appl. (1) 12 (1847), 265-290.

[80] Lytchak A., Thorbergsson G., Variationally complete actions on nonnegatively curved manifolds, Illinois J. Math. 51 (2007), 605-615.

[81] Magid M., Lorentzian isoparametric hypersurfaces, Pacific J. Math. 118 (1985), 165-197.

[82] Maxwell J.C., On the cyclide, Quart. J. of Pure and Appl. Math. 34 (1867), 111-126 (see also in Scientific papers, Vol. 2, Cambridge U. Press, 1890, 144-159).

[83] Milnor J., Morse theory, Ann. Math. Stud., Vol. 51, Princeton U. Press, Princeton, NJ, 1963.

[84] Miyaoka R., Compact Dupin hypersurfaces with three principal curvatures, Math. Z. 187 (1984), 433-452.

[85] Miyaoka R., Taut embeddings and Dupin hypersurfaces, in Differential Geometry of Submanifolds (Kyoto, 1984), Editor K. Kenmotsu, Lecture Notes in Math., Vol. 1090, Springer, Berlin - New York, 1984, 15-23.

[86] Miyaoka R., Dupin hypersurfaces and a Lie invariant, Kodai Math. J. 12 (1989), 228-256.

[87] Miyaoka R., Dupin hypersurfaces with six principal curvatures, Kodai Math. J. 12 (1989), 308-315. 
[88] Miyaoka R., The linear isotropy group of $G_{2} / S O(4)$, the Hopf fibering and isoparametric hypersurfaces, Osaka J. Math. 30 (1993), 179-202.

[89] Miyaoka R., The Dorfmeister-Neher's theorem on isoparametric hypersurfaces, math.DG/0602519.

[90] Miyaoka R., Ozawa T., Construction of taut embeddings and Cecil-Ryan conjecture, in Geometry of Manifolds, Editor K. Shiohama, Perspect. Math., Vol. 8, Academic Press, Boston, 1989, 181-189.

[91] Morse M., Cairns S., Critical point theory in global analysis and differential topology, Academic Press, New York, 1969.

[92] Mullen S., Isoparametric systems on symmetric spaces, in Geometry and Topology of Submanifolds VI, Editors F. Dillen et al., World Scientific, River Edge, NJ, 1994, 152-154.

[93] Münzner H.-F., Isoparametrische Hyperflächen in Sphären, Math. Ann. 251 (1980), 57-71.

[94] Münzner H.-F., Isoparametrische Hyperflächen in Sphären II: Über die Zerlegung der Sphäre in Ballbündel, Math. Ann. 256 (1981), 215-232.

[95] Niebergall R., Dupin hypersurfaces in $\mathbf{R}^{5}$. I, Geom. Dedicata 40 (1991), 1-22.

[96] Niebergall R., Dupin hypersurfaces in $\mathbf{R}^{5}$. II, Geom. Dedicata 41 (1992), 5-38.

[97] Niebergall R., Ryan P., Isoparametric hypersurfaces - the affine case, in Geometry and Topology of Submanifolds V, Editors F. Dillen et al., World Scientific, River Edge, NJ, 1993, 201-214.

[98] Niebergall R., Ryan P., Affine isoparametric hypersurfaces, Math. Z. 217 (1994), 479-485.

[99] Niebergall R., Ryan P., Focal sets in affine geometry, in Geometry and Topology of Submanifolds VI, Editors F. Dillen et al., World Scientific, River Edge, NJ, 1994, 155-164.

[100] Niebergall R., Ryan P., Affine Dupin surfaces, Trans. Amer. Math. Soc. 348 (1996), 1093-1117.

[101] Niebergall R., Ryan P., Real hypersurfaces in complex space forms, in Tight and Taut Submanifolds (Berkeley, CA, 1994), Editors T. Cecil and S.-S. Chern, Math. Sci. Res. Inst. Publ., Vol. 32, Cambridge Univ. Press, Cambridge, 1997, 233-305.

[102] Nomizu K., Some results in E. Cartan's theory of isoparametric families of hypersurfaces, Bull. Amer. Math. Soc. 79 (1973), 1184-1188.

[103] Nomizu K., Élie Cartan's work on isoparametric families of hypersurfaces, in Differential geometry (Stanford, 1973), Editors S.-S. Chern and R. Osserman, Proc. Sympos. Pure Math., Vol. 27, Amer. Math. Soc., Providence, RI, 1975, Part 1, 191-200.

[104] Nomizu K., On isoparametric hypersurfaces in Lorentzian space forms, Japan. J. Math. 7 (1981), $217-226$.

[105] Olmos C., Isoparametric submanifolds and their homogeneous structure, J. Differential Geom. 38 (1993), $225-234$.

[106] Ozawa T., On the critical sets of distance functions to a taut submanifold, Math. Ann. 276 (1986), 91-96.

[107] Ozeki H., Takeuchi M., On some types of isoparametric hypersurfaces in spheres. I, Tôhoku Math. J. 27 (1975), 515-559.

[108] Ozeki H., Takeuchi M., On some types of isoparametric hypersurfaces in spheres. II, Tôhoku Math. J. 28 (1976), 7-55.

[109] Palais R., Terng C.-L., A general theory of canonical forms, Trans. Amer. Math. Soc. 300 (1987), 771-789.

[110] Palais R., Terng C.-L., Critical point theory and submanifold geometry, Lecture Notes in Math., Vol. 1353, Springer, Berlin - New York, 1988.

[111] Peng C.-K., Hou Z., A remark on the isoparametric polynomials of degree 6, in Differential Geometry and Topology (Tianjin, 1986-87), Editors B. Jiang et al., Lecture Notes in Math., Vol 1369, Springer, Berlin New York, 1989, 222-224.

[112] Pinkall U., Dupin'sche Hyperflächen, Dissertation, Univ. Freiburg, 1981.

[113] Pinkall U., Letter to T. Cecil, December 5, 1984.

[114] Pinkall U., Dupin'sche Hyperflächen in $E^{4}$, Manuscripta Math. 51 (1985), 89-119.

[115] Pinkall U., Dupin hypersurfaces, Math. Ann. 270 (1985), 427-440.

[116] Pinkall U., Curvature properties of taut submanifolds, Geom. Dedicata 20 (1986), 79-83.

[117] Pinkall U., Thorbergsson G., Deformations of Dupin hypersurfaces, Proc. Amer. Math. Soc. 10 (1989), 1037-1043. 
[118] Pinkall U., Thorbergsson G., Examples of infinite dimensional isoparametric submanifolds, Math. Z. 205 (1990), 279-286.

[119] Pratt M.J., Cyclides in computer aided geometric design, Comput. Aided Geom. Design 7 (1990), 221-242.

[120] Pratt M.J., Cyclides in computer aided geometric design. II, Comput. Aided Geom. Design 12 (1995), 131-152.

[121] Reckziegel H., On the eigenvalues of the shape operator of an isometric immersion into a space of constant curvature, Math. Ann. 243 (1979), 71-82.

[122] Riveros C.M.C., Rodrigues L.A., Tenenblat K., On Dupin hypersurfaces with constant Möbius curvature, Pacific J. Math. 236 (2008), 89-103.

[123] Riveros C.M.C., Tenenblat K., On four dimensional Dupin hypersurfaces in Euclidean space, An. Acad. Brasil Ciênc. 75 (2003), 1-7.

[124] Riveros C.M.C., Tenenblat K., Dupin hypersurfaces in $\mathbf{R}^{5}$, Canad. J. Math. 57 (2005), 1291-1313.

[125] Rodrigues L.A., Tenenblat K., A characterization of Moebius isoparametric hypersurfaces of the sphere, Preprint, 2008.

[126] Ryan P.J., Hypersurfaces with parallel Ricci tensor, Osaka J. Math. 8 (1971), 251-259.

[127] Schrott M., Odehnal B., Ortho-circles of Dupin cyclides, J. Geom. Graph. 10 (2006), 73-98.

[128] Segre B., Una proprietá caratteristica de tre sistemi $\infty^{1}$ di superficie, Atti Acc. Sc. Torino 59 (1924), 666-671.

[129] Segre B., Famiglie di ipersuperficie isoparametrische negli spazi euclidei ad un qualunque numero di demesioni, Atti. Accad. naz Lincie Rend. Cl. Sci. Fis. Mat. Natur. 27 (1938), 203-207.

[130] Singley D., Smoothness theorems for the principal curvatures and principal vectors of a hypersurface, Rocky Mountain J. Math. 5 (1975), 135-144.

[131] Solomon B., The harmonic analysis of cubic isoparametric minimal hypersurfaces. I. Dimensions 3 and 6 , Amer. J. Math. 112 (1990), 157-203.

[132] Solomon B., The harmonic analysis of cubic isoparametric minimal hypersurfaces. II. Dimensions 12 and 24 , Amer. J. Math. 112 (1990), 205-241.

[133] Solomon B., Quartic isoparametric hypersurfaces and quadratic forms, Math. Ann. 293 (1992), $387-398$.

[134] Somigliana C., Sulle relazione fra il principio di Huygens e l'ottica geometrica, Atti Acc. Sc. Torino 54 (1918-1919), 974-979 (see also in Memorie Scelte, 434-439).

[135] Spivak M., A comprehensive introduction to differential geometry, Vol. 4, Publish or Perish, Boston, 1975.

[136] Srinivas Y.L., Dutta D., Blending and joining using cyclides, ASME Trans. J. Mechanical Design 116 (1994), 1034-1041.

[137] Srinivas Y.L., Dutta D., An intuitive procedure for constructing complex objects using cyclides, ComputerAided Design 26 (1994), 327-335.

[138] Srinivas Y.L., Dutta D., Cyclides in geometric modeling: computational tools for an algorithmic infrastructure, ASME Trans. J. Mechanical Design 117 (1995), 363-373.

[139] Srinivas Y.L., Dutta D., Rational parametrization of parabolic cyclides, Comput. Aided Geom. Design 12 (1995), 551-566.

[140] Stolz S., Multiplicities of Dupin hypersurfaces, Invent. Math. 138 (1999), 253-279.

[141] Strübing W., Isoparametric submanifolds, Geom. Dedicata 20 (1986), 367-387.

[142] Takagi R., A class of hypersurfaces with constant principal curvatures in a sphere, J. Differential Geom. 11 (1976), 225-233.

[143] Takagi R., Takahashi T., On the principal curvatures of homogeneous hypersurfaces in a sphere, in Differential Geometry (in Honor of Kentaro Yano), Kinokuniya, Tokyo, 1972, 469-481.

[144] Takeuchi M., Proper Dupin hypersurfaces generated by symmetric submanifolds, Osaka Math. J. 28 (1991), $153-161$.

[145] Takeuchi M., Kobayashi S., Minimal imbeddings of R-spaces, J. Differential Geom. 2 (1968), $203-215$.

[146] Tang Z.-Z., Isoparametric hypersurfaces with four distinct principal curvatures, Chinese Sci. Bull. 36 (1991), $1237-1240$.

[147] Tang Z.-Z., Multiplicities of equifocal hypersurfaces in symmetric spaces, Asian J. Math. 2 (1998), 181-214. 
[148] Terng C.-L., Isoparametric submanifolds and their Coxeter groups, J. Differential Geom. 21 (1985), 79-107.

[149] Terng C.-L., Convexity theorem for isoparametric submanifolds, Invent. Math. 85 (1986), 487-492.

[150] Terng C.-L., Submanifolds with flat normal bundle, Math. Ann. 277 (1987), 95-111.

[151] Terng C.-L., Proper Fredholm submanifolds of Hilbert space, J. Differential Geom. 29 (1989), 9-47.

[152] Terng C.-L., Recent progress in submanifold geometry, in Differential Geometry: Partial Differential Equations on Manifolds (Los Angeles, 1990), Editors R. Greene and S.T. Yau, Proc. Sympos. Pure Math., Vol. 54, Amer. Math. Soc., Providence, RI, 1993, Part 1, 439-484.

[153] Terng C.-L., Thorbergsson G., Submanifold geometry in symmetric spaces, J. Differential Geom. 42 (1995), $665-718$.

[154] Terng C.-L., Thorbergsson G., Taut immersions into complete Riemannian manifolds, in Tight and Taut Submanifolds, Editors T. Cecil and S.-S. Chern, Math. Sci. Res. Inst. Publ., Vol. 32, Cambridge Univ. Press, Cambridge, 1997, 181-228.

[155] Thorbergsson G., Dupin hypersurfaces, Bull. London Math. Soc. 15 (1983), 493-498.

[156] Thorbergsson G., Isoparametric foliations and their buildings, Ann. Math. 133 (1991), 429-446.

[157] Thorbergsson G., A survey on isoparametric hypersurfaces and their generalizations, in Handbook of Differential Geometry, Vol. I, Editors F. Dillen and L. Verstraelen, Elsevier Science, Amsterdam, 2000, 963-995.

[158] Töben D., Parallel focal structure and singular Riemannian foliations, Trans. Amer. Math. Soc. 358 (2006), 1677-1704, math.DG/0403050.

[159] Verhóczki L., Isoparametric submanifolds of general Riemannian manifolds, in Differential Geometry and Its Applications (Eger, 1989), Editors J. Szenthe and L. Tamássy, Colloq. Math. Soc. János Bolyai, Vol. 56, North-Holland, Amsterdam, 1992, 691-705.

[160] Wang C.-P., Surfaces in Möbius geometry, Nagoya Math. J. 125 (1992), 53-72.

[161] Wang C.-P., Möbius geometry for hypersurfaces in $S^{4}$, Nagoya Math. J. 139 (1995), 1-20.

[162] Wang C.-P., Möbius geometry of submanifolds in $S^{n}$, Manuscripta Math. 96 (1998), 517-534.

[163] Wang Q.-M., Isoparametric maps of Riemannian manifolds and their applications, in Advances in Science of China, Mathematics, Vol. 2, Editors C.H. Gu and Y. Wang, Wiley-Interscience, New York, 1986, 79-103.

[164] Wang Q.-M., Isoparametric functions on Riemannian manifolds. I, Math. Ann. 277 (1987), 639-646.

[165] Wang Q.-M., On the topology of Clifford isoparametric hypersurfaces, J. Differential Geom. 27 (1988), 55-66.

[166] West A., Isoparametric systems, in Geometry and Topology of Submanifolds, Editors J.-M. Morvan and L. Verstraelen, World Scientific, River Edge, NJ, 1989, 222-230.

[167] West A., Isoparametric systems on symmetric spaces, in Geometry and Topology of Submanifolds V, Editors F. Dillen et al., World Scientific, River Edge, NJ, 1993, 281-287.

[168] Wu B., Isoparametric submanifolds of hyperbolic spaces, Trans. Amer. Math. Soc. 331 (1992), 609-626.

[169] Wu B., A finiteness theorem for isoparametric hypersurfaces, Geom. Dedicata 50 (1994), $247-250$.

[170] Yau S.-T., Open problems in geometry, in Differential Geometry: Partial Differential Equations on Manifolds (Los Angeles, 1990), Editors R. Greene and S.T. Yau, Proc. Sympos. Pure Math., Vol. 54, Amer. Math. Soc., Providence, RI, 1993, Part 1, 439-484.

[171] Zhao Q., Isoparametric submanifolds of hyperbolic spaces, Chinese J. Contemp. Math. 14 (1993), 339-346. 